\title{
A REVIEW OF FLUID INSTABILITIES AND CONTROL STRATEGIES WITH APPLICATIONS IN MICROGRAVITY
}

\author{
J. Porter ${ }^{1,2, *}$, P. Salgado Sánchez ${ }^{1,2}$, V. Shevtsova ${ }^{3,4}$ \\ AND V. YASNOU ${ }^{3}$
}

\begin{abstract}
We give a brief review of several prominent fluid instabilities representing transitions driven by gravity, surface tension, thermal energy, and applied motion/acceleration. Strategies for controlling these instabilities, including their pattern formation properties, are discussed. The importance of gravity for many common fluid instabilities is emphasized and used to understand the sometimes dramatically different behavior of fluids in microgravity environments. This is illustrated in greater detail, using recent results, for the case of the frozen wave instability, which leads to large columnar structures in the absence of gravity. The development of these highly nonlinear states is often complex, but can be manipulated through an appropriate choice of forcing amplitude, container length and height, initial inclination of the surface, and other parameters affecting the nonlinear and inhomogeneous growth process. The increased opportunity for controlling fluids and their instabilities via small forcing or parameter changes in microgravity is noted.
\end{abstract}

Mathematics Subject Classification. 76E17, 76D33, 76E30.

Received October 7, 2020. Accepted March 17, 2021.

\section{INTRODUCTION}

The defining characteristic of fluids is the ease with which they can move in response to applied forcing or variations in external parameters. If the presence of forcing or a change in parameters causes one fluid configuration or dynamical state to lose stability to another, this can have important consequences. Fluids and their instabilities are a subject of extensive study in wide-ranging fields of physics, biology, engineering and medicine.

In large fluid systems, instabilities are frequently accompanied by questions of pattern selection arising from the competition among partially ordered states to replace a (typically) more homogeneous one. A classical example of this type of transition is the Turing instability [169], proposed in 1952 to explain how natural patterns

Keywords and phrases: Fluid instabilities, pattern selection, control, microgravity.

${ }^{1}$ E-USOC, Center for Computational Simulation, Universidad Politécnica de Madrid, Campus de Montegancedo, Boadilla del Monte, 28660 Madrid, Spain.

2 Escuela Técnica Superior de Ingeniería Aeronáutica y del Espacio, Universidad Politécnica de Madrid, Plaza Cardenal Cisneros 3, 28040 Madrid, Spain.

3 Microgravity Research Centre, CP-165/62, Université libre de Bruxelles (ULB), av. F. D. Roosevelt, 50 , 1050 Brussels, Belgium.

${ }^{4}$ Mechanical and Manufacturing Department, Mondragon Goi Eskola Politeknikoa (MGEP), Loramendi 4, Apdo. 23, 20500

Mondragon, Spain.

* Corresponding author: jeff.porter@upm.es 
such as stripes, hexagons, and spirals can arise in biological reaction-diffusion systems during morphogenesis. The selection of a final pattern after such an instability depends both on the linearized problem, which determines the properties of the unstable modes such as their characteristic lengthscale (wavelength), and the subsequent nonlinear evolution of the system, during which multiple unstable modes may interact and compete with each other.

For many applications, it is of considerable interest not only to understand, but to control the various instabilities that can occur. In the simplest scenario, one would like to maintain the stability of a desired fluid state and avoid parameters where that state loses stability to an undesired one. In other cases, one might want to trigger an instability at a particular time and to a state with particular properties. While there are nearly as many strategies for manipulating fluid states as there are fluid systems, we generalize here and identify three basic approaches to controlling them.

Control via initial conditions: Instabilities may be avoided or managed through judicial design of the fluid system and/or appropriately setting the initial conditions. Such an approach is particularly relevant for engineering applications where there is some flexibility in the design or setup. In principle, it requires obtaining a map of the relevant instability boundaries, either through experiment, theory, or simulation, and ensuring that operating parameters vary only within an acceptable range.

Control via forcing: If the instability arises from an external excitation of some kind, the character and magnitude of this forcing are crucial. By understanding how both the instability threshold and the properties of the unstable modes depend on the forcing, one can endeavor to control them. Supplemental forcing can also be added in an effort to modify or counteract the effects of the original one, either in a constant fashion, as with open-loop control, or with the use of feedback in a closed-loop control strategy, as discussed in [72].

Control via nonlinear evolution: In the case that an instability cannot be avoided or is called for by design, one can try to control the subsequent nonlinear evolution of the system in order to select a desired pattern. This could be done, for example, through boundary conditions, time-dependent control parameters [4], or by manipulating nonlinear interactions. With the Faraday instability, for instance, multifrequency forcing can be used to control nonlinear resonant triad interactions and, thereby, favor particular patterns $[123,131,166,167]$. Similar manipulation of three-wave interactions can be used to control the selection of patterns and dynamical states in coupled reaction-diffusion systems [27].

This paper provides a basic review of several prominent fluid instabilities and the strategies that have been used to control them. For simplicity, attention is restricted to simple single-component fluids. For example, instabilities associated with chemical reactions, phase changes, diffusion, or magnetohydrodynamics are only discussed in passing. Simple fluids of the type considered here possess four relevant types of energy: gravitational, chemical (in the broad sense), thermal, and kinetic. Numerous prominent instabilities involve a change in at least one of these energies, with the interaction among them determining the nature of the driving and restoring forces and, thus, the final state of the fluid.

The paper is organized as follows. Sections $2-5$ discuss common fluid instabilities associated with changes in gravitational, interfacial, thermal, and kinetic energy, respectively. Section 6 looks at the crucial role that gravity plays in many of these instabilities and the effect of reducing or eliminating it. Section 7 reviews some recent results on the frozen wave instability in more detail to illustrate the three general types of control strategies identified above. Conclusions are given in Section 8.

\section{InstaBiLity DUE TO GRAVITY}

Gravity is the dominant force affecting most fluid systems on earth. It drives the flow of water down streams and rivers and the eventual pooling of that water into lakes or oceans. It is a matter of common knowledge that if distinct fluids are placed together, the heavier ones sink while the lighter ones rise. In terms of energy, this occurs because gravity drives fluids to arrange themselves so that density contours align with those of the effective gravitational potential (the geoid). Locally, most large fluid interfaces are flat, with gravity acting to maintain them that way. 
If the initial state of a fluid system is gravitationally stable, then gravity plays the role of a restoring force. This is the case with gravity waves on the surface of a body of water, for example. On the other hand, if the density profile at some time is not gravitationally stable then the growth of perturbations that lower the total center of mass will be encouraged. The most basic gravitational instability of this type was described by Lord Rayleigh in 1882 [128], and later generalized to the case of accelerating fluids by G.I. Taylor [162].

\subsection{Rayleigh-Taylor instability}

The Rayleigh-Taylor instability is a mechanism by which gravitationally unstable configurations of fluids evolve toward a stable one. From a static fluid configuration, it can be observed when a heavier fluid lies above a lighter one, as with a layer of water above cooking oil. It also plays an important role in accelerating systems, such as the fronts resulting from combustion or explosions [24] and helps explain, for example, the mushroom clouds produced in a nuclear blast. It is important in the field of inertial confinement fusion [88].

An infinite flat interface separating a heavier fluid on the top from a lighter fluid on the bottom will always suffer a Rayleigh-Taylor instability. Indeed, if interfacial tension is neglected, all perturbation wavenumbers $k$ are unstable with a growth rate $s$ given by

$$
s^{2}=g \frac{\left(\rho_{1}-\rho_{2}\right)}{\left(\rho_{1}+\rho_{2}\right)} k
$$

where $g$ is the gravitational acceleration and $\rho_{1}$ and $\rho_{2}$ are the densities of the heavier and lighter fluids, respectively. If interfacial tension $\sigma$ is included, only perturbations with wavenumber less than $k_{c}=\sqrt{g\left(\rho_{1}-\rho_{2}\right) / \sigma}$ are unstable [12].

Figure 1 illustrates the type of behavior induced by the Rayleigh-Taylor instability, including mushroom-like caps that roll up at their edges. The instability in this case (see [173] for details) was induced by accelerating a container holding salt water (mixed with fluorescein dye) and heptane, which have a density ratio of $\rho_{2} / \rho_{1}=$ 0.497. The effective gravitational acceleration in the reference frame of the experiment was $0.32 \mathrm{~g}$ (upward).

\subsubsection{Strategies for control}

Since interfacial tension suppresses the Rayleigh-Taylor instability, inverted density profiles can be stabilized if the length of the interface is sufficiently small compared to $2 \pi / k_{c}$, such as in a thin straw holding water above air - of course, in this case, adhesive (capillary) forces are important as well.

It has been known for a long time that vibrations perpendicular to the interface can also suppress the Rayleigh-Taylor instability [175, 176], which is analogous to the stabilization of an inverted pendulum via the vertical vibration of its support [75]. If the eigenfrequencies of surface wave modes on the interface, which depend on its shape, are specified by $\Omega_{k}$, then applied vibration of amplitude $\varepsilon$ and frequency $\omega$ will stabilize the flat interface if

$$
\varepsilon \omega \gtrsim \frac{\sqrt{2} g}{\Omega_{k}}
$$

While the initial growth of unstable perturbations is exponential, they are soon affected by nonlinear interactions with each other and, eventually, with the system boundaries. In situations with $\rho_{1} \sim \rho_{2}$, the nonlinear growth tends to produce finger-like structures, often developing mushroom-like caps (see Fig. 1) while, if $\rho_{2} \ll \rho_{1}$, the preference is for bubble-like plumes or spikes [145]. If boundaries and densities can be varied, then the nonlinear growth of the pattern can be manipulated. 


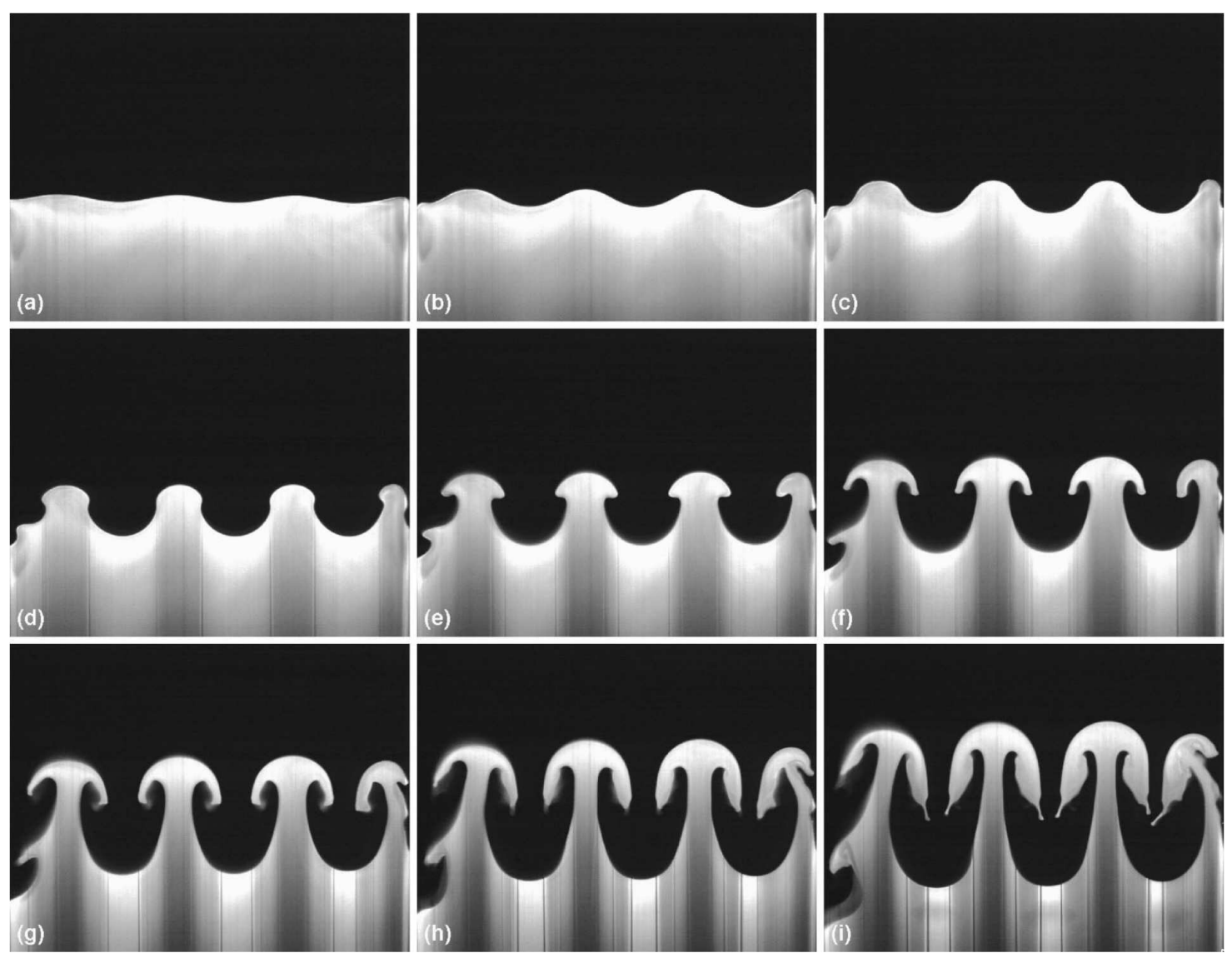

Figure 1. The Rayleigh-Taylor instability in an immiscible layer of salt water (mixed with fluorescein dye) and heptane, which have a density ratio of $\rho_{2} / \rho_{1}=0.497$. The effective gravitational acceleration is $0.32 \mathrm{~g}$ (upward). The images, which were taken with a separation of $0.05 \mathrm{~s}$, are produced using laser-induced fluorescence. Reprinted from Waddell et al. [173], with the permission of AIP Publishing.

\section{INSTABILITY DUE TO SURFACE TENSION}

Surface tension is the result of the cohesive forces acting between neighboring molecules in liquid. These intermolecular forces depend on the chemical composition and may involve hydrogen bonds (as with water), ionic bonding and/or van der Waals forces. The mutual attraction of molecules in a liquid means that those located along a surface or interface have a higher (intermolecular) energy, which causes that interface to behave as an elastic membrane attempting to minimize its area. This elastic force can support the weight of small non-wettable objects such as floating (waxy) leaves or water striders on the surface of a pond. With smaller liquid volumes (not dominated by gravity), surface tension favors drop-like shapes since, for an isolated free liquid of constant volume, the minimal surface is obtained for a sphere. In general, curvature induces a pressure jump across the interface described for static configurations by the Young-Laplace equation:

$$
\Delta p=-\sigma \nabla \cdot \hat{n}
$$

where $\Delta p$ is the pressure difference, $\sigma$ is the interfacial tension, and $\hat{n}$ is a unit normal vector. 


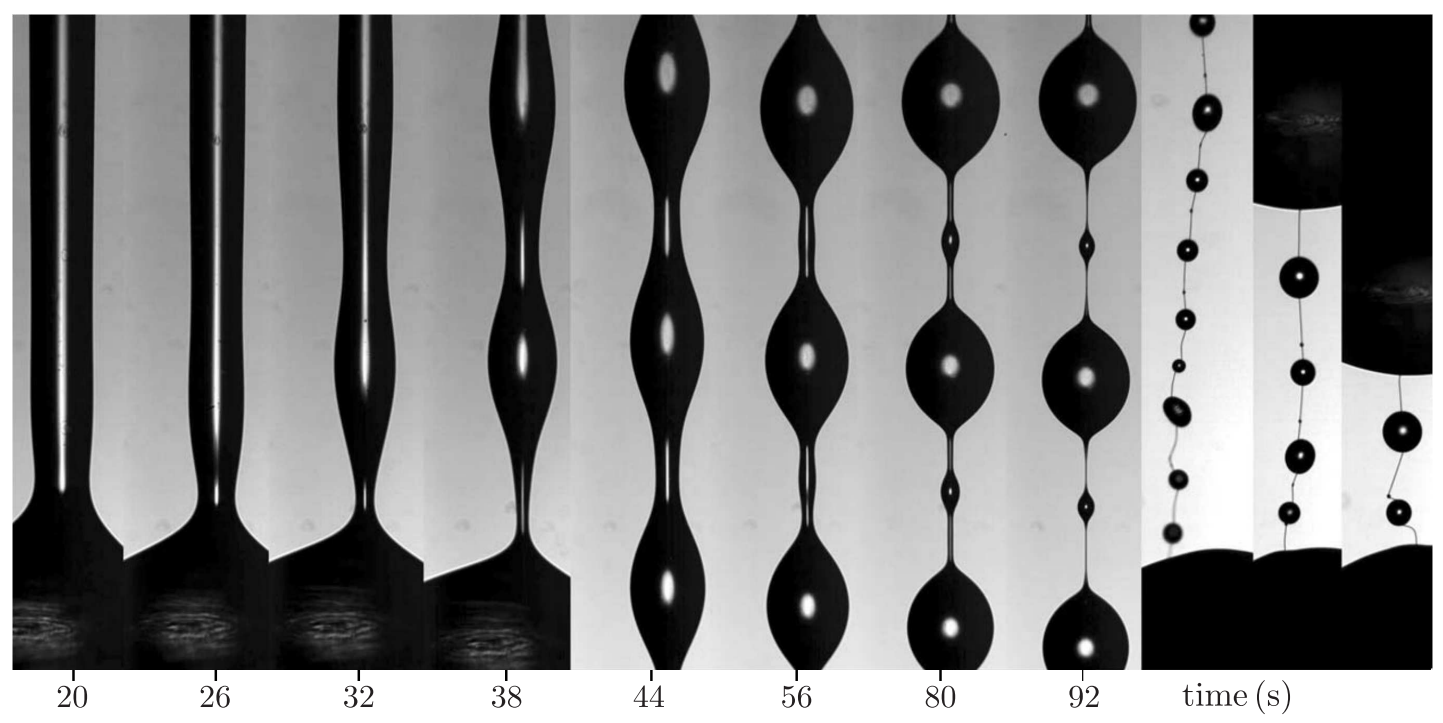

FiguRE 2. Plateau-Rayleigh type instability occurring on a filament of human saliva. The size of the images for $t<92 \mathrm{~s}$ is $0.21 \times 1.37 \mathrm{~mm}$. The final three images of size $0.42 \times 3.1 \mathrm{~mm}$ show the rupture of the droplets (connected by a polymeric fiber) initiated by agitating the system. Reprinted from Sattler et al. [141], with the permission of AIP Publishing.

Depending on boundary conditions, a fixed volume of liquid may have multiple configurations that are local minima (a single drop or two, for example), with each stable to small perturbations. A pure surface-tensiondriven instability occurs when there is a path between the current surface configuration and one of lower energy. The transition process is often abrupt and associated with the rupture (or merger) of one or more of the original surfaces.

\subsection{Plateau-Rayleigh instability}

The Plateau-Rayleigh instability [120, 130, 158] occurs when a column or stream of liquid breaks up into smaller drops, which have less total surface area. It can be observed in the dripping of a household tap and is involved in the formation of beads (dew drops) on a spider web. Among other applications, the instability is utilized in continuous ink jet technology [9] to generate a regular stream of small droplets. The development of this instability is illustrated in Figure 2 with an experiment (see [141] for details) beginning from a cylindrical filament of human saliva. Note that, in this case, the polymers contained in the fluid condense to leave a nearly solid fiber connecting the periodic series of droplets.

Linear stability analysis shows that a long cylindrical liquid column with radius $R$ is unstable to all perturbations of wavenumber $k<1 / R$, with the fastest growing one found at $k \simeq 0.697 / R$. While these values change for noncylindrical shapes [89] and if the liquid column makes contact with a solid structure, as in the case of a spider web, it is generally true that sufficiently long liquid columns are unstable to the formation of drops.

\subsubsection{Strategies for control}

Since the Plateau-Rayleigh instability occurs on long slender columns, stabilizing it requires limiting the length of the column or stream. A cylindrical liquid bridge, for example, will be stable in the absence of gravity if its length $L$ is less than the circumference $2 \pi R$ (equivalently, if its slenderness $S=L /(2 R)<\pi$ ). Gravity, which causes a vertical column to bulge near the bottom, affects these limits [155], as does contact with a solid support or substrate $[62,119]$. In fact, the growth of the instability may be arrested completely in the interior of a wetted cylindrical tube [37]. 
The maximum stable slenderness may be increased by the addition of external forcing in various ways. The shear force resulting from axial flow of an external fluid can have a stabilizing effect [90] as can thermocapillary flow [28] and vibration of the upper supporting disk [64]. Stabilization up to a slenderness of 4.3 (compared to $\pi$ for an unforced liquid bridge) can be achieved using acoustic radiation pressure with active feedback [104].

Since there is a band of unstable modes that may be selected through applied perturbations, there is considerable potential to control both their linear and nonlinear evolution and, thus, the final drop size and distribution. In applications that rely on the Plateau-Rayleigh instability to produce a uniform stream of drops, such as ink jet printers, their size and velocity is often selected using pressure or thermal perturbations of a particular frequency applied at the injection nozzle [105]. Satellite droplets (see Fig. 2 at $t=92 \mathrm{~s}$ ), which may form during the highly nonlinear breakup process, are a potential problem, and are typically managed through appropriate choice of forcing amplitude.

Furthermore, if two unstable modes are actively selected rather than one [36], their interaction can lead to velocity asymmetries in the drops produced by the instability. These initial drops may then coalescence in a regular fashion to produce a stream of larger, more widely spaced drops. Satellite droplets are effectively controlled with this method as well.

\section{INSTABILITY DUE TO THERMAL ENERGY}

Although purely thermal instabilities arise in some physical systems including interstellar gas clouds [23, 46], most fluid instabilities excited by thermal forcing occur through the temperature dependence of other fluid properties such as density or surface tension. Since most fluids expand with increasing temperature, nonuniform heating creates density gradients. Gravity then acts to align these gradients vertically and minimize the total gravitational potential energy. To the extent that hotter, lighter regions lie below cooler, denser regions, they experience a buoyant force. Furthermore, since surface tension generally depends on temperature, nonuniform heating also leads to tangential stress on a surface or interface as the regions of higher surface tension pull on the surrounding liquid with more force than those of lower surface tension.

\subsection{Rayleigh-Bénard convection}

Rayleigh-Bénard convection [13, 129] refers to the flow generated in a horizontal layer of fluid that is heated from below. It is a key feature of a wide range of geophysical, astrophysical, and engineering systems and a muchstudied experimental system due to its accessibility and the wide range of patterns that can be produced [68]. The instability results from the buoyancy of the hotter fluid, which is proportional to the temperature difference $\Delta T$ and the gravitational acceleration $g$. It is resisted by viscosity, which generates drag on the motion of fluid volumes with unequal density, and by thermal conductivity, which reduces their temperature and density difference. The dimensionless Rayleigh number,

$$
\mathrm{Ra}=\frac{\beta g H^{3} \Delta T}{\nu \alpha}
$$

characterizes the strength of the forcing. Here, $\beta$ is the volumetric thermal expansion coefficient, $\nu$ is the kinematic viscosity, $\alpha$ is the thermal diffusivity and $H$ is the height of the layer.

As Ra increases, the fluid transitions from a purely conductive motionless state to a pattern of convective cells. For an idealized configuration with two free boundaries, Lord Rayleigh [129] derived the critical value $\mathrm{Ra}_{c}=(27 / 4) \pi^{4} \simeq 657.5$. If the lower boundary is rigid, this threshold is given by $\mathrm{Ra}_{c} \simeq 1100.7$ while, with two rigid boundaries, it rises to $\mathrm{Ra}_{c} \simeq 1708$. The convection cells that emerge may be organized into rolls, squares, hexagons, or more complex patterns [21]. As Ra is increased further into the supercritical regime, the initial patterns can undergo a variety of additional instabilities [59] depending on fluid properties and boundary conditions. At sufficiently high values of Ra, turbulent flows predominate.

The theoretical stability criteria given above in terms of $\mathrm{Ra}_{c}$ depend on the Boussinesq approximation, which treats the fluid as incompressible except in the bouyancy term and assumes that its other physical properties 
are constant. Compressibility affects the threshold for convection as well as the patterns that develop afterward $[1,47,102,113]$. An alternative criterion [86] for instability in compressible fluids, which neglects viscosity and thermal conduction, is that the temperature gradient be larger than that corresponding to adiabatic equilibrium:

$$
-\frac{\mathrm{d} T}{\mathrm{~d} y}>\frac{\beta g T}{c_{p}}
$$

where $y$ is the vertical coordinate and $c_{p}$ is the specific heat at constant pressure. This condition is important, for instance, in atmospheric dynamics.

Some of the patterns that can be observed following the Rayleigh-Bénard instability are illustrated in Figure 3. These convective patterns (see [25] for details) were obtained in $\mathrm{CO}_{2}$ gas at a pressure of 41.59 bars in a square domain of width $63.2 \mathrm{~mm}$ with a fluid depth of $0.632 \mathrm{~mm}$. Each row shows two solutions obtained at the same Ra value but with different initial conditions, which demonstrates bistability.

\subsubsection{Strategies for control}

The most direct way to avoid Rayleigh-Bénard convection is to maintain Ra below the critical value. This depends, via equation (4.1), on the layer height, the viscosity, the thermal properties of the fluid, and the applied temperature difference $\Delta T$. Boundary conditions and compressibility further modify the instability limit.

Secondary forcing can also be used delay or manipulate the Rayleigh-Bénard instability. As with the Rayleigh-Taylor instability, high frequency vertical vibrations (in this case, parallel to the temperature gradient) can suppress convection [30,61, 160] as can modulation of the applied temperatures [31] or the addition of an oscillatory shear flow [76]. Closed-loop (feedback) control strategies have been investigated, with many based on the application of inhomogeneous perturbations to the heat flux at the lower boundary [67, 114]. Recently, for example, a reinforcement learning algorithm was shown to stabilize the Rayleigh-Bénard system at up to 30 times the critical $\mathrm{Ra}_{c}$ of the uncontrolled case [11].

\subsection{Thermocapillary convection}

Any variation in surface tension along a liquid/gas or liquid/liquid interface is associated with tangential stress since the liquid is pulled with greater force by the regions of higher surface tension. The resulting motion is referred to as the Marangoni effect [103] and, in the case that the surface tension gradients are the result of nonuniform temperature, is called thermocapillary (or Bénard-Marangoni) convection. The effect is important in numerous applications from bubbles [159] and foams to crystal growth [22], combustion [153], and heat transfer [137, 138].

Since temperature gradients generally induce variations in density (and other fluid properties) along with surface tension, thermocapillary convection is normally accompanied by natural (buoyant) convection, which, unless the layer in question is thin, is often stronger. When the influence of gravity is reduced, as in microgravity environments or at small scales, Marangoni effects may become the dominant source of fluid motion and have been studied in a series of liquid bridge experiments (see, e.g., the seminal investigations $[29,126,143,144]$ ) motivated by their relevance to the floating zone technique of crystal growth. Figure 4 illustrates thermocapillary flow in a liquid bridge of molten $\mathrm{NaNO}_{3}$ that is $5.4 \mathrm{~mm}$ long with a radius of $3.0 \mathrm{~mm}$. This flow, visualized with illuminated particles suspended in the liquid, is oscillatory with a period of approximately $2 \mathrm{~s}$ (see [126] for complete details). More recently, thermocapillary convection has been proposed as a mechanism to increase heat transfer and efficiency of phase change material (PCM) devices in microgravity applications [40, 41, 101, 137-139].

The strength of thermocapillary forcing is characterized by the dimensionless Marangoni number,

$$
\mathrm{Ma}=\frac{|\gamma| L \Delta T}{\mu \alpha}
$$



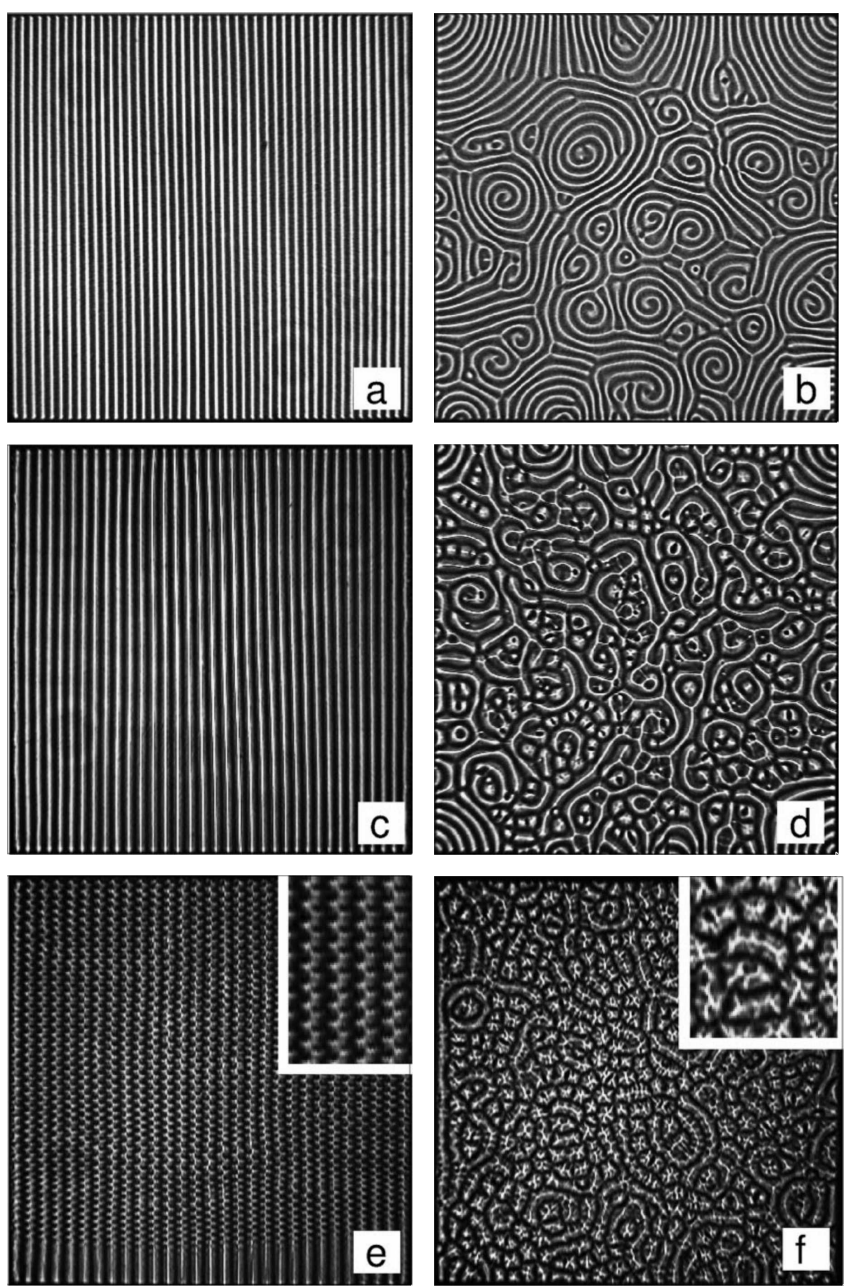

Figure 3. Patterns of Rayleigh-Bénard convection observed in a $0.632 \mathrm{~mm}$ deep layer of $\mathrm{CO}_{2}$ gas at a pressure of 41.59 bars in a square domain of width $63.2 \mathrm{~mm}$. Warm upflow occurs in the darker regions and cold downflow in the lighter ones. The reduced forcing $\epsilon=\left(\mathrm{Ra}-\mathrm{Ra}_{c}\right) / \mathrm{Ra}_{c}=$ 0.92 in (a) with straight roll solutions and in (b) with spiral defect chaos. The same types of solutions are seen in (c) and (d), respectively, with $\epsilon=2.99$ and $\epsilon=3.0$. Still further above onset with $\epsilon=5.08$, (d) oscillatory rolls and (f) oscillatory spiral defect chaos are found; insets show the rotating spoke patterns in these cases. Reprinted with permission from Cakmur et al. [25]. Copyright 1997 by the American Physical Society.

where $\mu$ is the dynamic viscosity and $L$ is the relevant length across which the temperature difference $\Delta T$ is applied. The thermocapillary coefficient $\gamma=\partial \sigma / \partial T$ describes the (linearized) dependence of surface tension $\sigma$ on temperature. As with Rayleigh-Bénard convection, the fluid motion is resisted by diffusion of temperature and momentum.

The case where the initial temperature gradient is perpendicular to the surface or interface, as with a flat horizontal layer of liquid heated from below, is qualitatively distinct from the more general case since there is no Marangoni effect associated with the initial state. It requires a critical $\Delta T$, corresponding to Ma $\simeq 80$ before convection occurs [116]. On the other hand, if there is a nonzero temperature gradient along the interface, then thermocapillary convection appears immediately [109]. 


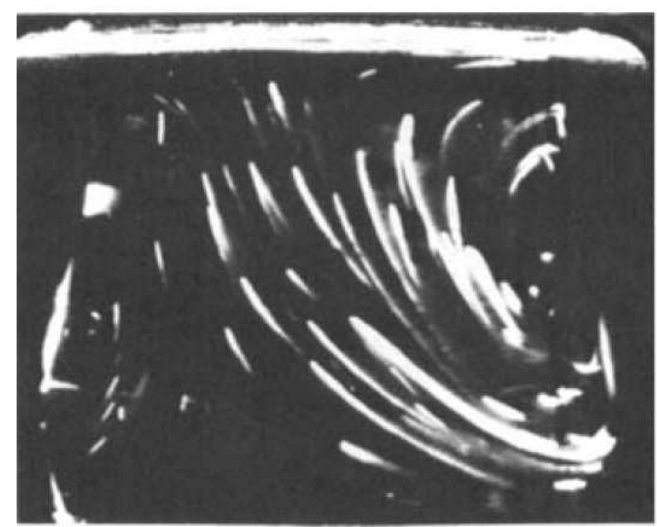

(a)

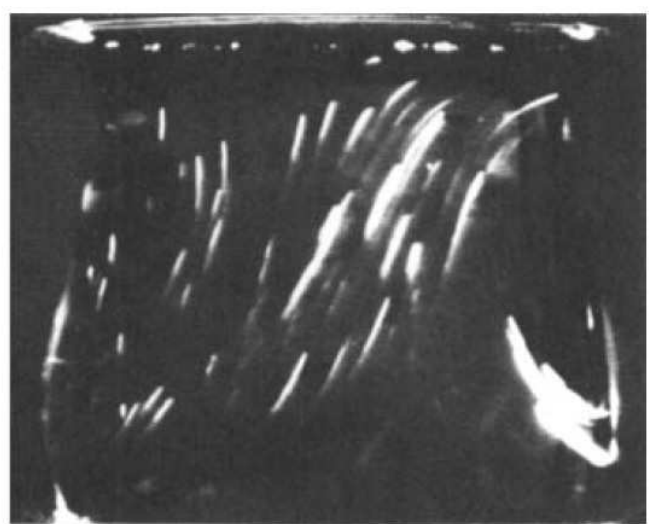

(b)

FIGURE 4. Thermocapillary flow in a half floating zone experiment (on ground) with molten $\mathrm{NaNO}_{3}$, which has a Prandtl number of 8.9. The liquid bridge is $5.4 \mathrm{~mm}$ long with a radius of $3.0 \mathrm{~mm}$ (slenderness $S=0.9$ ). The flow, which is visualized by tracking illuminated particles suspended within the melt, is oscillatory. The two images in (a) and (b) are separated by approximately $1 \mathrm{~s}$, which is half of the oscillation period. Reproduced with permission from Preisser et al. [126], where a complete description of the experiment can be found.

\subsubsection{Strategies for control}

The most direct way to control thermocapillary convection is to maintain Ma below its critical value. In the case of horizontal temperature gradients, the critical value of greatest interest is that associated with the transition from steady to oscillatory convection. With the floating zone technique of crystal growth, for example, the excitation of oscillatory modes is responsible for unwanted striations in impurity or dopant concentration [74]. A review of control strategies for the floating zone technique is provided by Lappa [87].

For a given applied temperature, the strength of the Marangoni forcing can be controlled by manipulating surface properties and, in particular, the coefficient $\gamma$. With a molten silicone liquid bridge, for example, an increase in oxygen partial pressure of the surrounding argon gas was shown to stabilize steady thermocapillary flow [6] and attributed to a decrease in $|\gamma|$ due to the presence of a $\mathrm{SiO}_{2}$ film. More generally, surfactants can have a significant effect on the thermocapillary flow [15, 20,79] and may be stabilizing or destabilizing.

Various types of additional forcing have been investigated for their ability to control thermocapillary convection including magnetic fields [57, 174], heat flux modulation [107] and vibrations [2, 91, 92, 100, 163, 178]. Another approach $[35,52,81,147,148,177]$ is to impose a particular flow of gas along the thermocapillary surface, which both provides a viscous shear that can oppose the Marangoni shear force and modifies the heat exchange between liquid and gas, which affects the temperature distribution along the surface.

Control strategies utilizing feedback have also been investigated; see, e.g., References [7, 112, 140]. In the case of a half-zone liquid bridge, the onset of oscillatory convection may be delayed by more than $40 \%$ through appropriate placement of sensors and heaters to modify the surface temperature [151].

\section{INSTABILITy DUE TO MOTION/ACCELERATION}

A number of the most common fluid instabilities are driven by the transfer of kinetic energy, either through the movement of an immersed solid or boundary, or from regular motion already present in the underlying state. In some contexts, these are called inertial or dynamic instabilities. Common everyday examples include the generation of waves on the ocean by wind, whirlpools in the wake of a moving boat, and the waves that radiate outward from a stone tossed into a quiet pond. 
When the inciting motion is not overly disruptive, both gravity and surface tension are expected to act as restoring forces. In the example of a stone disturbing the surface of a quiet pond, the waves eventually dissipate due to viscosity (and collisions with the shore) and the water returns to a quiescent state. Just as with Rayleigh-Bénard or thermocapillary convection, such instabilities can only be sustained with the continued input of energy. In laboratory experiments, this is often accomplished with regular periodic motion such as (linear) vibration or rotation.

In this section we describe several common phenomena associated with shear flow or vibrational forcing (see also the book by Lyubimov et al. [97]) including Faraday waves, cross-waves, Kelvin-Helmholtz waves, frozen waves and vibroequilibria. In each of these cases, both gravity and surface tension act to constrain the growth of the excited states. The absence of strong gravity can, therefore, have a dramatic effect both on the threshold (if present) of the instability and on the subsequent nonlinear growth of unstable modes. After discussing the contrasting features of these instabilities in terrestrial and microgravity environments in Section 6, we focus on the frozen wave instability, in particular, and illustrate several strategies for controlling it with a summary of recent experimental and numerical results in Section 7.

\subsection{Directly forced waves}

If an object is dipped into a fluid and moved back and forth, as a spoon in a cup of coffee, it naturally generates waves with the same frequency, a response that is both familiar and easy to understand. More generally, when a fluid is driven by a moving object or boundary, its reaction reflects the properties of that excitation.

Resonant waves of this type are deliberately generated in wavemaker experiments [63] and can be controlled through the selection of amplitude and frequency. Spurious waves, appearing with frequencies other than the desired one, can be reduced with appropriate corrections of the wavemaker motion $[78,142]$ or with feedback. At lower frequencies, when the wavelength is comparable to the container size, the fluid motion may be described as sloshing; see [42], for example, for an overview of this topic.

Resonant (harmonic) waves are almost always present when a fluid is excited by vibrations or oscillatory motion of some kind. There is no threshold to be overcome since the forcing is direct, i.e., the fluid must move in reaction to the forcing no matter how small its amplitude is. A notable exception to this is when the entire fluid is moved as a solid body. This is the case for the idealized Faraday experiment, discussed below, which is not associated with harmonic surface waves. In other systems, the harmonic wave motion may be localized to the region near the moving object or boundary; these are sometimes referred to as meniscus waves when the motion is vertical [33].

\subsection{Parametrically forced waves}

An everyday example often used to illustrate parametric forcing is the pumping of a swing, which can be done by raising the center of mass at the lowest point, decreasing its distance from the pivot, then extending the separation again at the highest points, where the vertical component of this action is less. The difference in work done by these two acts provides a net input of energy that increases the swinging motion (or maintains it against damping). Two things about this simple mechanical example should be noted. First, the forcing is faster than the response, with half its period. Second, the stable unforced equilibrium, with the swing (pendulum) hanging straight down is not immediately affected by the forcing, which adds no net energy from that equilibrium state.

Parametrically forced waves arise through analogous mechanisms when the oscillation of a parameter does not directly destroy the original stable state, but does provide a means to increase the energy of small perturbations at the resonant frequencies. Typically, the strongest response is the subharmonic one, at half the forcing frequency. In real fluid systems with damping, a finite critical forcing amplitude is required to observe the instability. In most cases, the forcing is applied through vibrations of part or all of the solid structure.

\subsubsection{Faraday waves}

In the many years that have passed since Faraday's seminal work [43], subharmonic waves excited by vertical vibration have received a great deal of attention (see, e.g., Refs. [3, 14, 39, 82, 108]) due to their convenient 
size and timescale for laboratory experiments and the many interesting patterns that can be produced. In the standard setup, an open container of fluid is shaken up and down while boundary effects like meniscus waves are suppressed or ignored. Just as in the example of pumping a swing, this vertical motion of the center of mass does not disrupt the initial flat surface but does provide a mechanism for destabilizing resonant perturbations. The system is typically analyzed in an accelerating reference frame attached to the container, where the applied forcing manifests as a modulation of the effective gravity and, hence, of the natural frequency of gravity-capillary waves.

The Faraday wave threshold for immiscible fluid layers can be determined from a Floquet analysis of the linearized Navier-Stokes equations [85]. If viscosity is ignored, the instability boundaries for the free surface of a liquid are described by the well-known Mathieu equation [14]. If the damping is finite but small, and the liquid layer is assumed to be deep compared to the wavelength of the excited waves, then a quasi-potential approximation can be used to derive amplitude equations [179] describing the behaviour of Faraday waves near onset. In this limit, the linear damping arises only from bulk dissipation and the threshold is determined by a damped Mathieu equation [85].

The critical acceleration $a$ needed to excite subharmonic waves on the interface between two immiscible fluids satisfies [170]

$$
a \simeq 8 \omega k \frac{\mu_{1}+\mu_{2}}{\rho_{1}-\rho_{2}},
$$

where $\rho_{1}$ and $\rho_{2}<\rho_{1}$ are the densities of the liquids, $\mu_{1}$ and $\mu_{2}$ are their dynamic viscosities, and $k$ is the subharmonic wavenumber, which is related to the frequency of vibration $2 \omega$ via the dispersion relation

$$
\omega^{2}=\frac{\rho_{1}-\rho_{2}}{\rho_{1}+\rho_{2}} g k+\frac{\sigma}{\rho_{1}+\rho_{2}} k^{3}
$$

Note that the critical acceleration $a$ is proportional to the product of viscosity, frequency and wavenumber. Both gravity $g$ and surface (interfacial) tension $\sigma$ act as restoring forces that limit the magnitude of the excited waves. However, for a given forcing frequency $2 \omega$, the wavenumber $k$ satisfying equation (5.2) decreases upon increasing either $g$ or $\sigma$, which means that the critical forcing of equation (5.1) also decreases. Due to the dependence of dissipation on wavenumber, both restoring forces are actually destabilizing for the onset of Faraday waves.

\subsubsection{Strategies for control of Faraday waves}

Clearly, Faraday waves can be avoided by remaining below threshold, which depends on vibration frequency and on viscosity. In small or shallow containers, this threshold also depends on the damping in the boundary layers and on the modified wavenumber selection induced by these boundaries. In fact, since shallow layers suppress long wavelengths due to greater interaction with the viscous boundary layer at the bottom, they can favor resonant modes at higher $k$, including harmonic oscillations [84].

When the wavelength is not sufficiently small compared to the lateral boundaries, Faraday waves interact with these boundaries, which affects the final pattern. Circular containers, for example, can be used to produce patterns with $n$-fold rotational symmetry [39, 146], while (small) square or rectangular containers promote different modes and dynamics $[110,152]$ consistent with that geometry.

Since the frequency content of the forcing function selects resonant wavenumbers via the dispersion relation, manipulation of this forcing function provides considerable control over the Faraday waves that appear. Monochromatic sinusoidal forcing can be used to excite a particular wavenumber, while two-frequency forcing can be used to excite two distinct modes, which then interact. Superlattice or quasicrystalline structures can be produced in this way $[3,38,39,83,167]$, along with a rich variety of other patterns. A modulated (drifting) subharmonic superlattice solution obtained using two-frequency forcing with $40 \mathrm{~Hz}$ and $70 \mathrm{~Hz}$ is shown in Figure 5; see [3] for details of that experiment. 

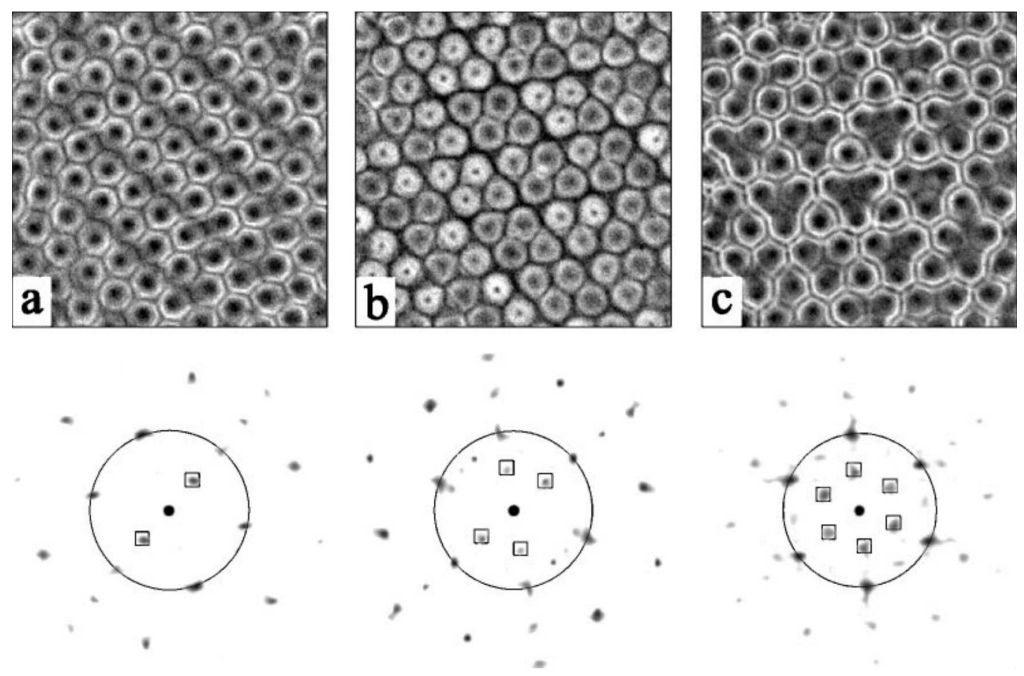

FIgURE 5. Subharmonic superlattice (type I) state with broken symmetry obtained using twofrequency forcing of $40 \mathrm{~Hz}$ and $70 \mathrm{~Hz}$ with no phase offset in a $1.55 \mathrm{~mm}$ deep layer of $23 \mathrm{cSt}$ silicone oil. The region shown is $9 \mathrm{~cm}$ squared. The circles in the power spectra (bottom row) indicate the wavevectors of the primary hexagon mode. The solution drifts slowly between the three patterns $(\mathrm{a}-\mathrm{c})$ over 10-60 s. Reprinted with permission from Arbell and Fineberg [3]. Copyright 2002 by the American Physical Society.

Multifrequency forcing with more than two components offers still more possibilities $[32,123,166]$ as the various forcing ratios and phase differences come into play. In fact, it is not even necessary to (linearly) excite additional modes for them to have an important effect on the nonlinear interactions of the primary mode. Damped modes have been shown to have a significant effect on pattern selection [123, 154, 166, 167].

\subsubsection{Cross-waves}

The cross-wave instability is commonly observed in wavemaker experiments $[8,108]$ when sufficient forcing is applied. As indicated by its name, the instability produces waves that are oriented (nearly, but not completely $[117,118,121])$ perpendicular to the forcing axis and to the directly forced harmonic waves. They were first described by Faraday [43], in an experiment with a partially submerged vibrating plate, as forming a series of apparently permanent ridges projecting outward "like the teeth of a coarse comb". Like Faraday waves, cross-waves are typically subharmonic and often grow quickly to large amplitude, obscuring the initial harmonic wave field. They are easily produced by partially or entirely submerged vibrating objects and by oscillating boundaries $[10,43,80,111,161]$.

Theoretically, one of the simplest systems in which to study cross-waves is a horizontally vibrated container, which can be treated in the comoving frame of the container using a time-dependent body force (an oscillating horizontal component of effective gravity). In this case, each of the endwalls acts as a wavemaker producing harmonic waves and, with sufficient forcing, cross-waves. Garrett [55] was the first to show that the onset of the cross-wave instability could be described by Mathieu's equation and, thus, is parametric in nature. Further progress was made after Jones [71] derived a nonlinear Schrödinger equation valid in the limit of weak damping and weak forcing. The parametric forcing mechanism of cross-waves, in contrast to the uniform vertical forcing of Faraday waves, is inhomogeneous and concentrated near the endwalls [118, 165, 172].

The threshold for cross-waves strongly depends on the properties, motion, and depth of the wavemaker producing them - generally, the deeper the wavemaker (or the liquid layer in the case of a horizontally vibrated container), the more fluid is displaced and the lower the amplitude needed to observe the instability. The energy 


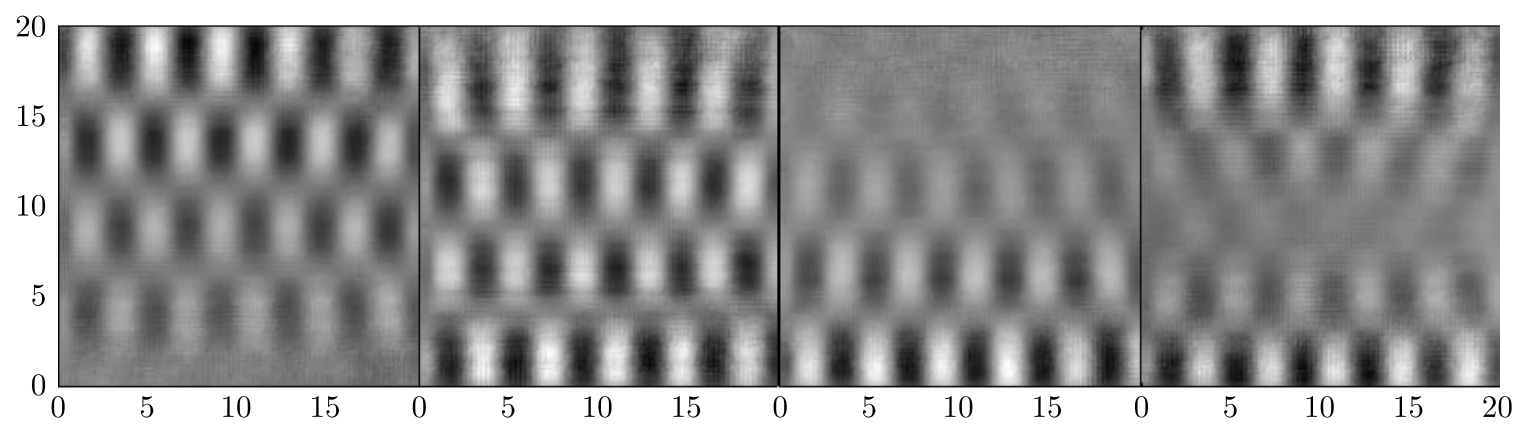

Figure 6. Modulated (quasiperiodic) cross-wave solution found in a $20 \mathrm{~cm}$ square container (distance marked along the axes) holding $5 \mathrm{cSt}$ oil and vibrated horizontally (up and down in the figure) at $13.95 \mathrm{~Hz}$. The period of modulation is approximately $45.6 \mathrm{~s}$ and the snapshots, with time increasing left to right, are separated by approximately $1 / 8$ of this period. See Tinao et al. [165] for more details.

balance for sufficiently high frequencies is similar to that of Faraday waves [see Eq. (5.1)], with the critical forcing increasing with viscosity and with forcing frequency (wavenumber) [118, 121, 165].

\subsubsection{Strategies for control of cross-waves}

Cross-waves can be avoided by remaining below the critical amplitude which, as noted above, depends mainly on the viscosity, forcing frequency, and the properties of the wavemaker. In comparison to vertically forced Faraday waves in large aspect ratio containers, it is more difficult to control cross-wave patterns after excitation. This is primarily due to the influence of the localized forcing mechanism, which is strongest along the wavemaker (or endwall) and decays with increasing distance from it [172]. Crosswise oriented standing waves, evanescent in the downstream direction, reflect the crosswise orientation of this supercritical (strongly forced) region. Although mode interactions [5, 165] and secondary instabilities [171] occur, there is less flexibility for controlling pattern selection than in the Faraday wave system.

An interesting exception to this occurs in horizontally vibrated containers when the wave trains emanating from opposite sides interact in the interior. Since the forcing at the two endwalls is out of phase by $\pi$, the subharmonic waves they produce are out of phase by $\pi / 2$. This phase difference leads to a primary Hopf bifurcation and correspondingly modulated solutions $[118,122,135,165]$. The modulation frequency is controlled by the strength of the interaction and, thus, by the damping, forcing frequency and container length. By selecting frequency and detuning (in the crosswise direction), one can control the nature and extent of these modulated solutions and produce patterns that cycle back and forth from one side to the other with variable period. The primary Hopf bifurcation as well as the saddle-node heteroclinic bifurcation that destroys the modulated states is well-captured by a coupled oscillator model [135], which suggests that a rich variety of additional dynamics can be selected by varying the forcing and container size. Figure 6 shows a cross-wave solution obtained experimentally by controlling, via the forcing frequency, the strength of interaction in the interior. The region of dominant excitation cycles between one side and the other (upper and lower boundaries in the figure) with a period of $45.6 \mathrm{~s}$.

\subsection{Kelvin-Helmholtz instability}

The Kelvin-Helmholtz instability [65, 77] occurs when there is both a density gradient and a sufficiently large velocity gradient within a fluid. It is a relatively common phenomenon in nature that lies behind a class of wind-driven water waves and billow (Kelvin-Helmholtz) clouds. It is important in plasma physics and inertial confinement fusion in particular. The structures that develop from the instability, which may rapidly become turbulent, play a crucial role in the transfer of energy from larger to smaller scales. 

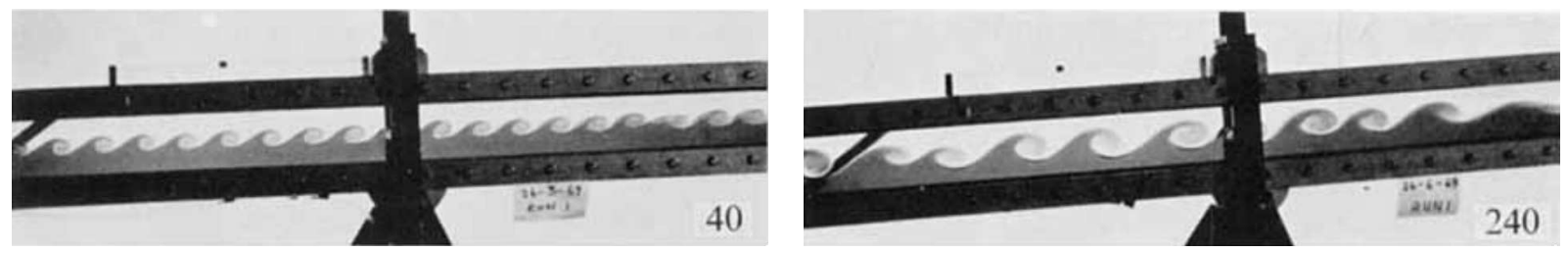

FIGURE 7. Snapshots showing the miscible interface between brine and water after the KelvinHelmholtz instability. The angle of inclination controls the velocities of the underlying flow while the effective thickness of the interface is controlled by the time of diffusion, shown in minutes by the label at the lower right. Reproduced with permission from Thorpe [164], where a full description of the experiment can be found.

Figure 7 shows two pictures from an experiment (see [164] for details) that initiated the Kelvin-Helmholtz instability by inclining a plexiglass tube containing brine and water. The waves that develop in this case, on a miscible interface, depend on its effective thickness, which is controlled by the time of diffusion. These Kelvin-Helmholtz patterns show the typical roll-up of the wave tips caused by the shear flow.

The physical source of the instability is the underlying shear flow and its kinetic energy. An exchange of fluid between faster and slower moving regions may result in a reduction of this kinetic energy and, when the difference is greater that any associated increase in gravitational energy, it can drive the growth of perturbations [34]. This balance between buoyancy and the available kinetic energy in the shear flow is characterized by the Richardson number Ri, which may be defined locally as

$$
\mathrm{Ri}=-\frac{g(d \rho / \mathrm{d} y)}{\rho(d V / \mathrm{d} y)^{2}}
$$

where $y$ is the vertical coordinate and $\rho$ and $V$ are the local density and velocity, respectively. For an incompressible inviscid fluid, a necessary condition for instability is that $\mathrm{Ri}<1 / 4$.

The Kelvin-Helmholtz instability produces waves that are (approximately) stationary with respect to the mean flow and is typical of systems in which the characteristic thickness of the density profile is comparable to that of the velocity profile. On the other hand, if the region with a large density gradient is more localized than the shear layer, the flow can be unstable to cusp-like traveling waves called Holmboe modes [66, 124, 156, 157]. These waves have opposite propagation speeds (in a reference frame moving with the mean flow) and can lead to the ejection of interfacial fluid from the crest into the upper and lower layers [26].

The theoretical analysis of the Kelvin-Helmholtz instability is simplified if the density gradient and shear are both localized to an interface separating two distinct fluids. Consider, for example, two layers of immiscible liquids of equal height $H$, each initially moving uniformly in the horizontal $x$ direction but at different velocities, $V_{1}$ and $V_{2}$. Assuming this configuration is gravitationally stable (no Rayleigh-Taylor instability) and neglecting viscosity, perturbations of wavenumber $k$ will grow when the velocity difference $V=\left|V_{1}-V_{2}\right|$ satisfies [48]

$$
V^{2}=\frac{\left(\rho_{1}+\rho_{2}\right)}{\rho_{1} \rho_{2}}\left[\sigma k+\frac{\left(\rho_{1}-\rho_{2}\right) g}{k}\right] \tanh (k H)
$$

where $\rho_{1}$ and $\rho_{2}<\rho_{1}$ are (as before) the lower and upper fluid densities, respectively, and $\sigma$ is the interfacial tension. Note that, in the absence of interfacial tension, large wavenumbers $(k \rightarrow \infty)$ are always unstable.

If the fluid layers are sufficiently deep, with $H>H^{*}=\left[3 \sigma /\left(\rho_{1}-\rho_{2}\right) g\right]^{1 / 2}$, then the minimum of equation (5.4) occurs at finite $k$. In the limit $H \gg H^{*}$, this critical wavenumber $k_{c}$ is given by the inverse of the capillary 
length:

$$
k_{c}=\sqrt{\frac{\left(\rho_{1}-\rho_{2}\right) g}{\sigma}} .
$$

With expression (5.5) and $k H \gg 1$, the critical velocity difference $V_{c}$ can be obtained explicitly as

$$
V_{c}^{2}=\frac{2\left(\rho_{1}+\rho_{2}\right)}{\rho_{1} \rho_{2}} \sqrt{\sigma g\left(\rho_{1}-\rho_{2}\right)}
$$

Note that gravity, whose influence is proportional to the density difference, and interfacial tension are the determining factors for both the critical velocity difference $V_{c}$ and the wavenumber $k_{c}$ of the corresponding unstable mode. Viscosity has an effect [48], but is not essential to the onset of the instability.

\subsubsection{Strategies for control}

The Kelvin-Helmholtz instability can be avoided by maintaining $\mathrm{Ri}$ in the stable region $(>1 / 4)$ or reducing the velocity difference across the interface in question. Both gravity and interfacial tension are stabilizing, but additional forcing can also be used for control. In the context of plasmas, for example, both electric [70] and magnetic fields [125] have been shown to suppress the instability. Stabilization via feedback has been investigated as well $[115,127]$.

\subsubsection{Frozen wave instability}

The classical Kelvin-Helmholtz instability driven by a steady shear tends to produce waves that curl and roll up (see Fig. 7) into a series of vortices or "cat-eye" structures. Such flows are highly nonlinear and can quickly become turbulent. A variation of the Kelvin-Helmholtz mechanism produced by an oscillatory base flow avoids this complication since the average shear force is zero, which reduces the tendency for roll-up and leads to more regular waves with a time-averaged left-right reflection symmetry. Indeed, this reflection symmetry helps explain why the instability produces standing or "frozen" waves rather than traveling waves.

The theory of frozen waves was developed following Lyubimov and Cherepanov [93] by assuming two infinitely extended layers of immiscible fluids initially separated by a flat interface in a gravitationally stable configuration. If this system is subjected to horizontal vibrations with a frequency $\omega$ and amplitude $A$, then the inviscid limit admits a counterflow solution with the layers uniformly oscillating back and forth and out of phase with each other. If the layers are of the same height $H$ and there is no net volume flux, then the velocities $V_{j}$ in each layer are of the form

$$
V_{j}(t)=\frac{\rho_{1}-\rho_{2}}{\rho_{1}+\rho_{2}}(-1)^{j} v \sin (\omega t), \quad j=1,2,
$$

where $v=A \omega$. Under the assumption that the forcing frequency $\omega$ is much greater than the inverse of the characteristic viscous time, a separation of timescales approach can be applied. This counterflow then affects the slower growth of the frozen wave instability through a time-averaged quadratic nonlinearity. Replacing the $V^{2}$ in equation (5.4) by $\left\langle\left(V_{1}-V_{2}\right)^{2}\right\rangle=2 v^{2}\left(\rho_{1}-\rho_{2}\right)^{2} /\left(\rho_{1}+\rho_{2}\right)^{2}$, where the angled brackets denote a time average, one obtains the forcing threshold for frozen waves of wavenumber $k$ :

$$
v^{2}=\frac{\left(\rho_{1}+\rho_{2}\right)^{3}}{2 \rho_{1} \rho_{2}\left(\rho_{1}-\rho_{2}\right)^{2}}\left[\sigma k+\frac{\left(\rho_{1}-\rho_{2}\right) g}{k}\right] \tanh (k H) .
$$


As with the classical Kelvin-Helmholtz instability, the most unstable wavenumber in the limit of deep layers is given by equation (5.5). The corresponding threshold is

$$
v_{c}^{2}=\frac{\left(\rho_{1}+\rho_{2}\right)^{3}}{\rho_{1} \rho_{2}\left(\rho_{1}-\rho_{2}\right)} \sqrt{\frac{\sigma g}{\rho_{1}-\rho_{2}}} .
$$

In the case of thin layers with $H<H^{*}$, the minimum of the neutral stability curve occurs at $k=0$, corresponding to longwave perturbations. These extended modes grow above a critical forcing velocity of

$$
v_{c}^{2}=\frac{\left(\rho_{1}+\rho_{2}\right)^{3} g H}{2 \rho_{1} \rho_{2}\left(\rho_{1}-\rho_{2}\right)} .
$$

Lyubimov and Cherepanov [93] also performed a weakly-nonlinear analysis to obtain an expression for the (peak-to-peak) amplitude $h$ of the frozen wave pattern (see also [49]) in the case of deep layers with $k_{c}$ given by equation (5.5):

$$
h^{2}=\frac{16 \rho_{1}\left(\rho_{1}+\rho_{2}\right) \sqrt{\sigma\left(\rho_{1}-\rho_{2}\right)}}{\rho_{2}\left(42 \rho_{1} \rho_{2}-11 \rho_{1}^{2}-11 \rho_{2}^{2}\right)} g^{-3 / 2}\left(v^{2}-v_{c}^{2}\right) .
$$

Equation (5.11) predicts a supercritical bifurcation to stable small-amplitude waves when $\rho_{2} / \rho_{1}>0.2829$, which holds for most practical combinations of liquids. This supercritical bifurcation was confirmed experimentally by Jalikop and Juel [69] and more recently, by the numerical work of Gligor et al. [60].

Figure 8 shows the frozen wave instability observed on the interface between Galden HT135 and $200 \mathrm{cSt}$ silicone oil with forcing velocity $v$ between $0.30 \mathrm{~m} / \mathrm{s}$ and $0.58 \mathrm{~m} / \mathrm{s}$ (see [69] for details). The densities of these liquids are $1752 \mathrm{~kg} / \mathrm{m}^{3}$ and $962 \mathrm{~kg} / \mathrm{m}^{3}$, respectively, and the interfacial tension is $6.8 \mathrm{mN} / \mathrm{m}$. Over the range of forcing applied, the frozen waves develop from small sinusoidal disturbances to larger inverted trochoid or finger-like shapes.

The frozen wave instability will be considered in more detail in Section 7 along with several strategies for controlling it in the context of microgravity.

\subsection{Vibroequilibria}

Most vibrations, transmitted by moving boundaries or immersed solids, for example, produce inhomogeneous oscillatory velocity fields - one exception is the idealized Faraday system below threshold, where the vibrations are assumed to be both uniform and parallel to any density gradients (perpendicular to the initial interface). An inhomogeneous velocity field is associated with a dynamic pressure gradient that alters the average equilibrium position of any fluid with a free interface. Although this type of deformation was observed by Faraday [43] in his experiments on the flattening of fluid drops suspended beneath a vibrating plate, in most typical situations the effect is negligible compared to gravity and surface tension. In microgravity environments, on the other hand, the vibroequilibria effect can be significant, particularly if surface (interfacial) tension is also weak or absent, as it is with miscible liquids.

The theory describing these quasi-static equilibrium states, which are known as vibroequilibria, was developed using a time-averaged variational approach $[16,17,56,94,95]$ that is valid in the limit of inviscid fluid, irrotational flow, and a forcing frequency that is large compared to that of the primary sloshing mode. In this case, the time-averaged surface or interface can be obtained by minimizing an energy functional (an averaged Lagrangian) that takes into account gravitational, surface, contact, and vibrational energy. All possible fluid configurations must be considered, and multiple local minima may coexist [45]. Already qualitatively consistent with early experiments $[19,50,175,176]$, the predictions of vibroequilibria theory were recently confirmed by quantitative comparison with simulations of the Navier-Stokes equations [45] and with the results of a microgravity experiment specifically dedicated to observing the effect [44, 132]. 


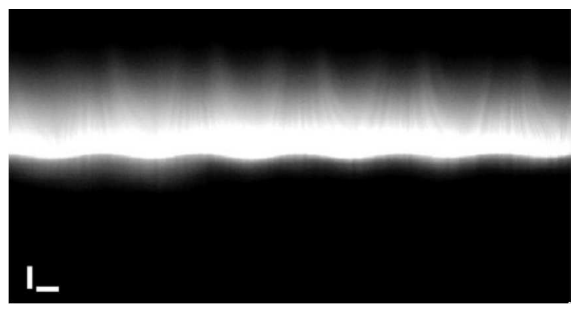

(a)

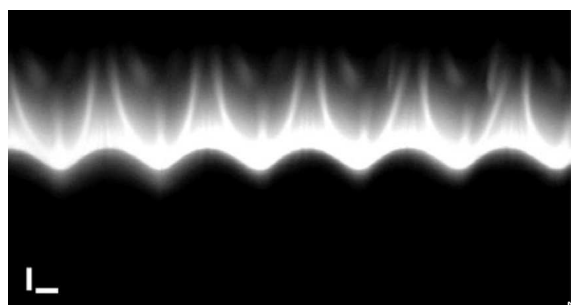

(b)

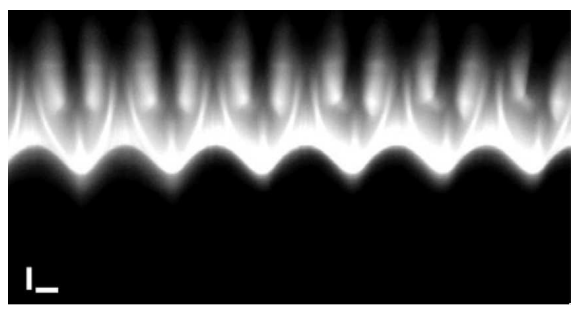

(c)

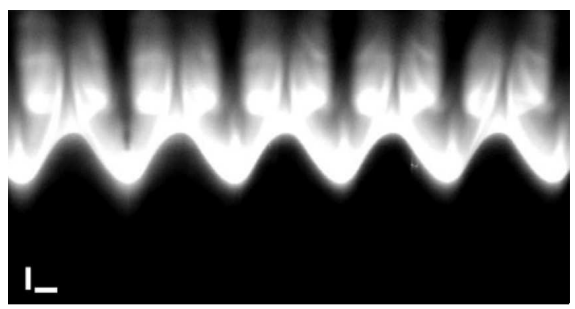

(d)

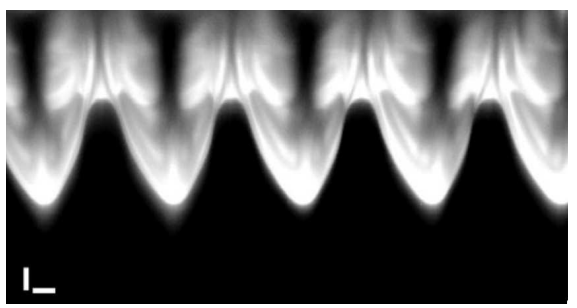

(e)

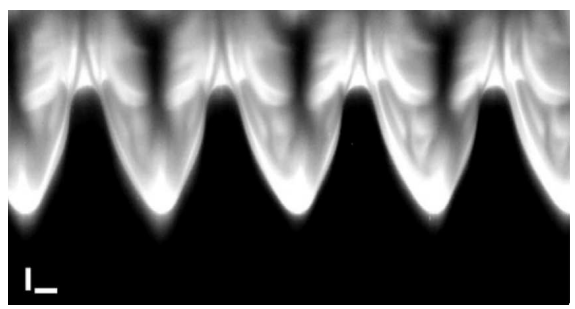

(f)

FiguRE 8. The frozen wave instability on the interface between Galden HT135, with density $\rho_{1}=1752 \mathrm{~kg} / \mathrm{m}^{3}$, and $200 \mathrm{cSt}$ silicone oil, with density $\rho_{2}=962 \mathrm{~kg} / \mathrm{m}^{3}$. The interfacial tension is $6.8 \mathrm{mN} / \mathrm{m}$. The forcing velocity $v(\mathrm{in} \mathrm{m} / \mathrm{s}$ ) takes increasing values: (a) 0.30 (b) 0.34 (c) 0.38 (d) 0.45 (e) 0.54 (f) 0.58 . The short white lines (lower left corners) indicate the capillary length of $0.94 \mathrm{~mm}$. Reproduced with permission from Jalikop and Juel [69], where a full description of the experiment can be found.

\subsubsection{Strategies for control}

The vibroequilibria effect depends both on the shape and size of the container holding the fluid and on the nature of the vibrational forcing. In the limit of large amplitude, the effect is analogous to gravity [18] in the sense that density contours tend to align perpendicular to the forcing axis (assuming linear motion). Flat interfaces can be stabilized by vertical (perpendicular) vibrations and are destabilized by horizontal (parallel) vibrations, which cause the fluid to move upward along the lateral walls $[45,56,132]$. This behaviour offers the possibility for the vibroequilibria effect to be used to control the distribution of fluids, particularly in microgravity where small-amplitude vibrations may be sufficient.

The potential for controlling liquids in microgravity was directly investigated by the "Control of Fluids in microgravity with Vibrations" (CFVib) experiment [45, 132], which used piezoelectric ceramics and a cantilever configuration to vibrate cylindrical and cuboidal containers. A selection of the experimental results from that parabolic flight experiment is reproduced in Figure 9, which clearly demonstrates the vibroequilibria phenomenon in a cylindrical container holding silicone oil. The large back-to-front component of the vibrations causes the oil to climb the front and rear sides while dipping in the interior. In general, the results of the experiment confirm the predicted gravity-like tendency of vibroequilibria solutions to align large portions of the average surface so as to be more perpendicular to the vibrational axis [132]. 


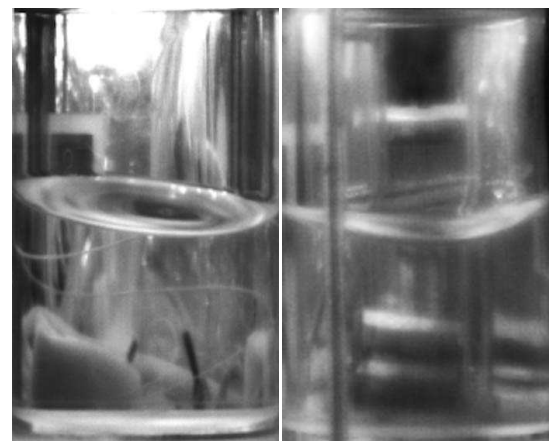

(a) $t=0 \mathrm{~s}$

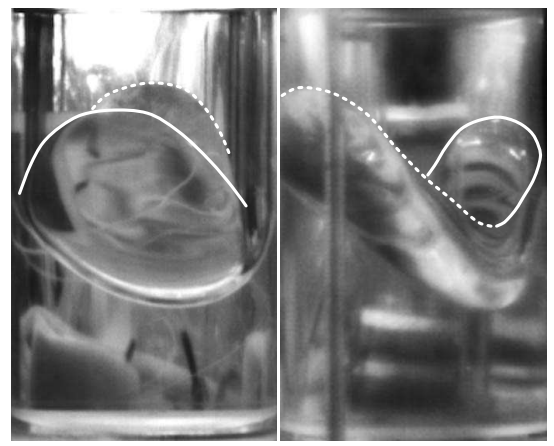

(b) $t=2.021 \mathrm{~s}$

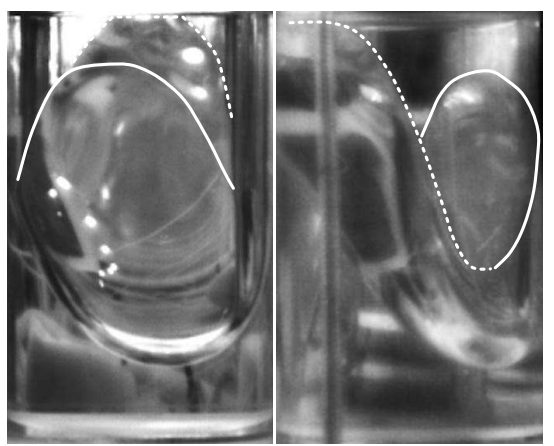

(c) $t=2.492 \mathrm{~s}$

Figure 9. The vibroequilibria effect in microgravity. Front and lateral views of $50 \mathrm{cSt}$ silicone oil (with air above) in a cylindrical container of height $60 \mathrm{~mm}$ and interior radius $15 \mathrm{~mm}$ vibrated at $f=59.3 \mathrm{~Hz}$ with a vertical velocity component $v_{z}$ of $94.8 \mathrm{~mm} / \mathrm{s}$. The labeled observation times are given with respect to the initiation of the forcing in microgravity: (a) $t=0 \mathrm{~s}$, (b) $t=2.021 \mathrm{~s}$, (c) $t=2.492 \mathrm{~s}$. The front and rear contact lines are outlined with solid and dashed curves, respectively. Details of the experiment can be found in [45, 132].

Even in the case that the vibroequilibria effect is not large, it may have a significant influence on other instabilities. An example of this will be discussed in Section 7, where the vibroequilibria effect both modifies the underlying equilibrium state and represents a type of (average) force that affects the growth of unstable modes following the frozen wave instability.

\section{EFFECT OF GRAVITY ON INSTABILITIES}

Gravity plays a key role in the basic fluid instabilities discussed in Sections 2, 4, and 5, either as a driving force, with the Rayleigh-Taylor and Rayleigh-Bénard instabilities, or as a restoring force, with interfacial gravity-capillary waves of all types and with vibroequilibria. Low gravity or weightless environments are thus characterized both by the reduction or absence of some instabilities, like natural (buoyant) convection, and by significant changes in the threshold and in the nonlinear growth and pattern selection properties of other instabilities.

Even prior to the consideration of instabilities, gravity has a profound effect on the underlying equilibrium state. In microgravity environments, the preference for flat surfaces and flat density contours, which are assumed properties of the base state in most theoretical analyses, is lost. In its place, surface tension favors minimumsurface configurations that are generally curved (perfectly spherical drops, for example), while contact forces can lead the fluid to fully cover adjacent solid boundaries. The appearance of more complex curved interfaces obscures the separation between fluid phenomena associated with vertical (perpendicular) forcing, such as Faraday waves, and those associated with horizontal (tangential) forcing, like frozen waves and cross-waves.

As gravitational energy becomes negligible compared to surface (interfacial) and contact energies, there is less resistance to large-scale motion and changes in shape. Relatively small vibrations, often called g-jitter in microgravity environments and arising from on-board machinery, crew movements, orbit or docking maneuvers, and so on, can have an amplified effect. It is important, for one thing, to understand exactly what effects are expected from vibrations in order to avoid or minimize disruption to sensitive experiments [73]. Although the g-jitter present in space environments is largely unwelcome, deliberately applied vibrations can mimic the effects of gravity, as mentioned in Section 5.4, and may thus be useful in some situations. For instance, vibrations can induce convection patterns $[54,58,106,150]$ similar to Rayleigh-Bénard (buoyancy driven) convection in a fluid confined between two plates held at different temperatures. The fact that the remaining restoring forces, like surface tension, are often small and place no real limitations on changes in the center of mass of the fluid 


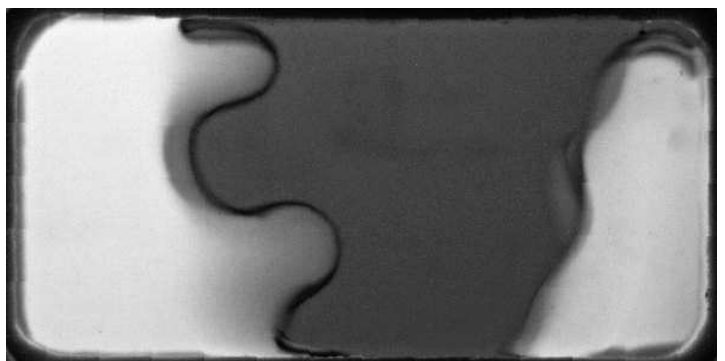

(a)

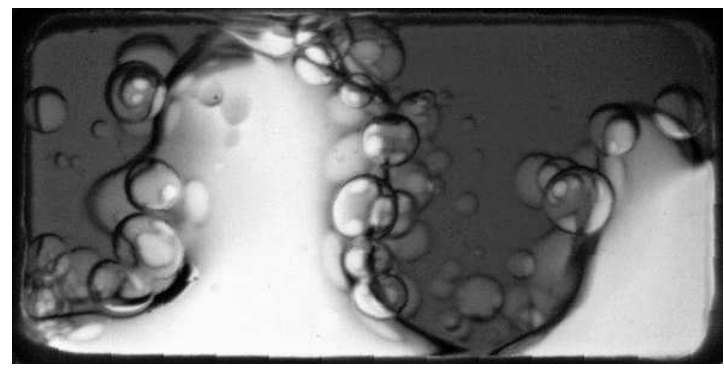

(b)

FIGURE 10. Interface between FC-40 $\left(\rho_{1}=1855 \mathrm{~kg} / \mathrm{m}^{3}\right.$, lighter shade) and silicone oil (darker shade) after the frozen wave instability in a container with $L=15 \mathrm{~mm}$ and $2 H=7.5 \mathrm{~mm} \mathrm{during}$ a parabolic flight experiment (see [136] for details). (a) $20 \mathrm{cSt}$ silicone oil $\left(\rho_{2}=950 \mathrm{~kg} / \mathrm{m}^{3}\right.$ ) in microgravity with $22 \mathrm{~Hz}$ vibrations at $A=1.76 \mathrm{~mm}(v=0.243 \mathrm{~m} / \mathrm{s})$, showing secondary Faraday waves. (b) $2 \mathrm{cSt}$ silicone oil $\left(\rho_{2}=950 \mathrm{~kg} / \mathrm{m}^{3}\right)$ in hypergravity with $50 \mathrm{~Hz}$ vibrations at $A=3.9 \mathrm{~mm}(v=1.225 \mathrm{~m} / \mathrm{s})$, showing Faraday waves and drops.

explains why small-amplitude vibrations, among other strategies, have the potential to manipulate and control fluids in low gravity environments.

The more rapid growth of instabilities in the absence of a gravitational restoring force and the increased potential for interaction between them will be illustrated in more detail in the case of the frozen wave instability, introduced in Section 5.3.2. The appearance of this instability in microgravity is associated with behavior that differs markedly from the finite-amplitude (supercritical) waves observed in normal gravity conditions (see Fig. 8). For one thing, the threshold of longwave perturbations vanishes in the inviscid large-aspect-ratio limit [see Eq. (5.10)]. Wavenumber selection within the band of unstable modes is then controlled by a combination of growth rate [99] and finite-size effects [134]. The curvature of the underlying surface, amplified by the vibroequilibria effect, means that the frozen waves are generally not of uniform amplitude across the interface. Furthermore, they grow rapidly, quickly transforming into nonlinear columnar structures in many cases $[51,98,99,134,136,149]$. Because such columnar structures effectively rotate large portions of the interface into a nearly perpendicular orientation with respect to the forcing, they can support Faraday waves. Finally, drop ejection may occur as well if these secondary Faraday waves are of sufficiently large amplitude. Figure 10 illustrates this complicated type of dynamics, observed during a parabolic flight experiment (see [136] for more details).

\section{EXAMPLE: Frozen WAVE INSTABILITY IN MICROGRAVITY}

As mentioned above, the frozen wave instability leads to very different patterns in microgravity. Not only do frozen waves appear at much lower forcing values, but they grow rapidly into large columnar structures that interact both with the container length and with its height $[53,134]$. They develop in a nonuniform fashion that depends on the way in which the forcing is initiated, often moving from the lateral walls inward. Mode competition and defect formation are common. Moreover, since the final state is composed of a series of nearly vertical interfaces separating successive columns, it is susceptible to secondary instabilities including Faraday waves [99, 149] and, beyond that, drop ejection [136].

Several of these complicating factors are anticipated in the results of classical frozen wave theory, which is discussed in the following section. Sections 7.2-7.4 then consider the effects of forcing, initial conditions, and nonlinear growth in the selection of the final fluid configuration and the ways in which these things can be used to control the development of the instability. 


\subsection{Theoretical predictions}

The ideal inviscid theory for infinitely extended fluid layers that is summarized in Section 5.3.2 provides the threshold, critical wavenumber, and the branching coefficient that characterizes the (typically supercritical) growth of frozen waves as forcing is increased. It is evident from those results that gravity plays an essential role in each of these properties. Substituting $g=0$ into equation (5.8) shows that the minimum of the neutral stability curve occurs at $k=0$ and that there is no longer any critical forcing required to excite this extended mode [see also Eq. (5.10) with $g=0$ ]. Consequently, any finite forcing $v>0$ will excite a band of unstable modes $0 \leq k \leq k_{m}$ with the wavenumber $k_{m}$ of the neutrally stable mode determined by the equation

$$
k_{m} \tanh \left(k_{m} H\right)=\frac{2 \rho_{1} \rho_{2}\left(\rho_{1}-\rho_{2}\right)^{2}}{\sigma\left(\rho_{1}+\rho_{2}\right)^{3}} v^{2} .
$$

The fact that there is a band of unstable modes complicates the pattern selection problem. Lyubimova et al. [99] dealt with this by assuming that the fastest growing mode would eventually dominate and maximizing growth rate over the interval $0 \leq k \leq k_{m}$. The most dangerous wavenumber $k^{*}$ was found to satisfy

$$
k^{*}\left[\tanh \left(k^{*} H\right)+\frac{k^{*} H}{3 \cosh ^{2}\left(k^{*} H\right)}\right]=\frac{4 \rho_{1} \rho_{2}\left(\rho_{1}-\rho_{2}\right)^{2}}{3 \sigma\left(\rho_{1}+\rho_{2}\right)^{3}} v^{2}
$$

in the infinitely extended inviscid case, which was then compared with numerical simulations and experiments. It was found that, while viscosity can have a significant effect, there is a monotonic increase of pattern wavenumber with applied forcing, a tendency that was also confirmed by Salgado Sanchez et al. [134]. Indeed, for $k^{*} H>1$, the relationship is nearly quadratic, with $k^{*} \simeq 2 k_{m} / 3 \propto v^{2}$. This simple prediction that larger forcing leads to patterns with larger wavenumbers is modified by finite-size effects, as discussed in Section 7.2.

Gravity also provides a critical restoring force that limits the amplitude of unstable perturbations. The nonlinear branching coefficient of equation (5.11) relating the squared wave amplitude to (reduced) forcing diverges with decreasing gravity as $g^{-3 / 2}$. This indicates that for low gravity levels, frozen waves grow very rapidly in amplitude when the forcing surpasses the critical value. The nature of this transformation from a supercritical pitchfork bifurcation to a (perturbed) degenerate pitchfork bifurcation as the gravity level decreases was recently examined by Gligor et al. [60].

\subsection{Effect of the forcing}

The primary effect of the forcing amplitude in microgravity is to select the wavenumber of the frozen wave patterns, as indicated by equation (7.2). This is illustrated in Figure 11, which shows the evolution of the interface separating immiscible liquids corresponding to the fluorocarbon oil FC-40 and $20 \mathrm{cSt}$ silicone oil, which have densities $\rho_{1}=1855 \mathrm{~kg} / \mathrm{m}^{3}$ and $\rho_{2}=950 \mathrm{~kg} / \mathrm{m}^{3}$, respectively, and an interfacial tension $\sigma$ of approximately $6 \mathrm{mN} / \mathrm{m}$. The simulations, details of which can be found in [134], are performed in a rectangular container of length $L=30 \mathrm{~mm}$ and height $2 H=7.5 \mathrm{~mm}$.

The difference between the two simulations in Figure 11 is the magnitude of the forcing velocity, which may be measured in terms of the dimensionless vibrational parameter

$$
\mathcal{F}=v \sqrt{\frac{\left(\rho_{1}-\rho_{2}\right) L}{\sigma}}
$$

that arises after scaling distance with $L$, density with $\left(\rho_{1}-\rho_{2}\right)$ and time with $\sqrt{\left(\rho_{1}-\rho_{2}\right) L^{3} / \sigma}$. The simulations in columns (a) and (b) of Figure 11 correspond to $\mathcal{F}=28.48$ and 37.97, respectively. The snapshots in this figure show the mean interface and velocity field (indicated with scaled arrows) obtained by averaging over one period of excitation. The harmonic surface waves, which are always present due to the direct forcing mechanism described in Section 5.1, are filtered out by this averaging. 
(a) $\mathcal{F}=28.48$
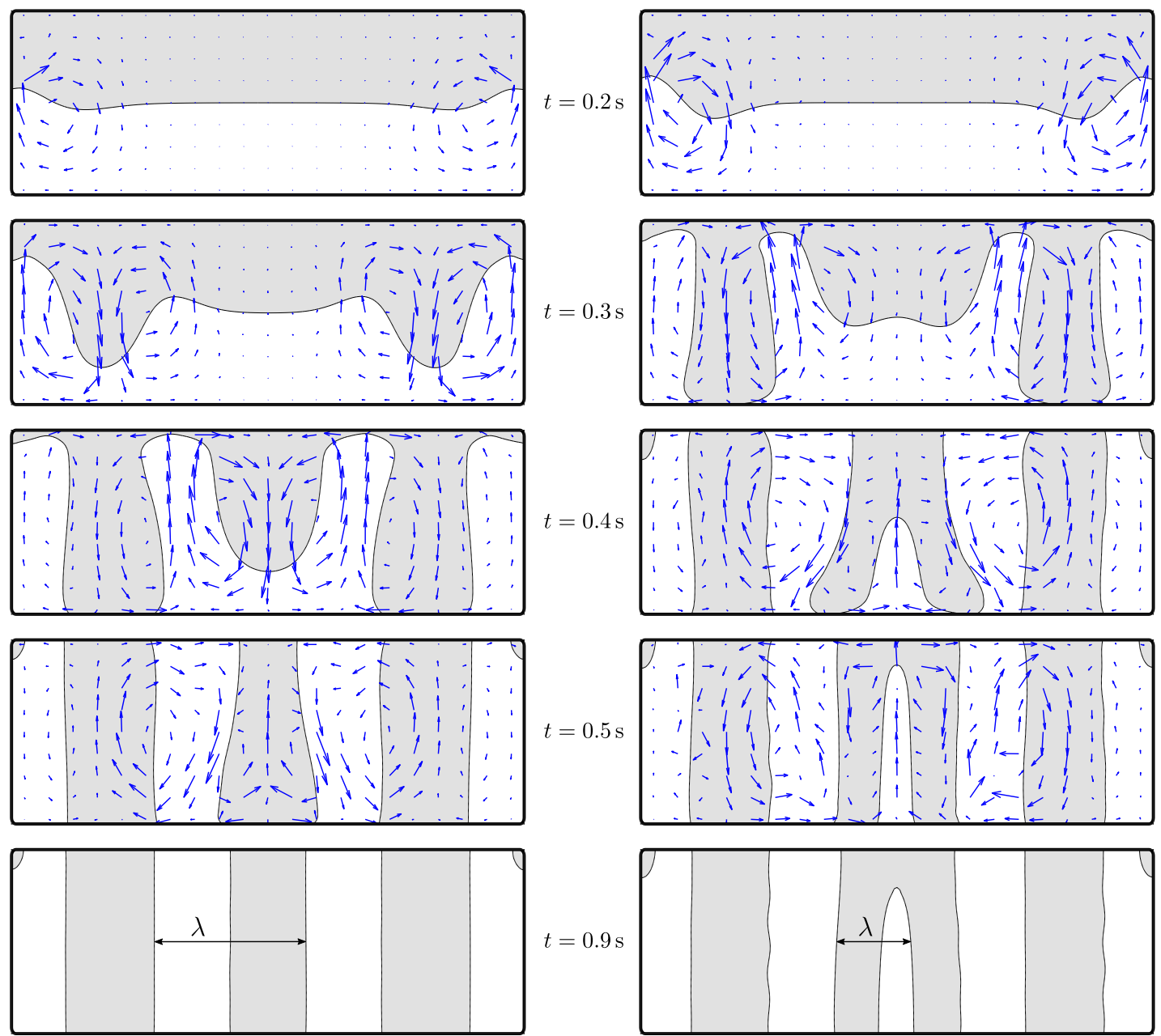

FIgURE 11. Evolution of the interface, averaged over one excitation period, after the frozen wave instability in weightless conditions. Simulations (see [134] for details) are performed with parameters appropriate to FC-40 and $20 \mathrm{cSt}$ silicone oil in a container with $L=30 \mathrm{~mm}$ and $2 H=7.5 \mathrm{~mm}$ vibrated with (a) $\mathcal{F} \simeq 28.48$ and (b) $\mathcal{F} \simeq 37.97$. The evolution time of the snapshot is indicated between the columns while the mean velocity field is shown using scaled arrows (scale not preserved between snapshots). The measurement of the local wavelength $\lambda$ in the defect region (used below) is illustrated for the final state $(t=0.9 \mathrm{~s})$. Subharmonic Faraday waves on the lateral boundaries of the columnar structure can be observed in (b).

Note that the wavenumber of the pattern in the simulation with lower amplitude $\mathcal{F}=28.48$ is also lower, with the final state composed of three column pairs of approximately equal width (identifying left and right boundaries). The simulation with $\mathcal{F}=37.97$ has a larger average wavenumber with a smaller incomplete column (or defect) in the center. The formation of these defects, which gradually grow into new columns as $\mathcal{F}$ increases, is investigated in $[134,168]$ and will be discussed further in Section 7.4. For low forcing amplitudes, the new columns tend to appear in the central region of the container while, for larger amplitude, they appear off-center, in an asymmetric fashion. 


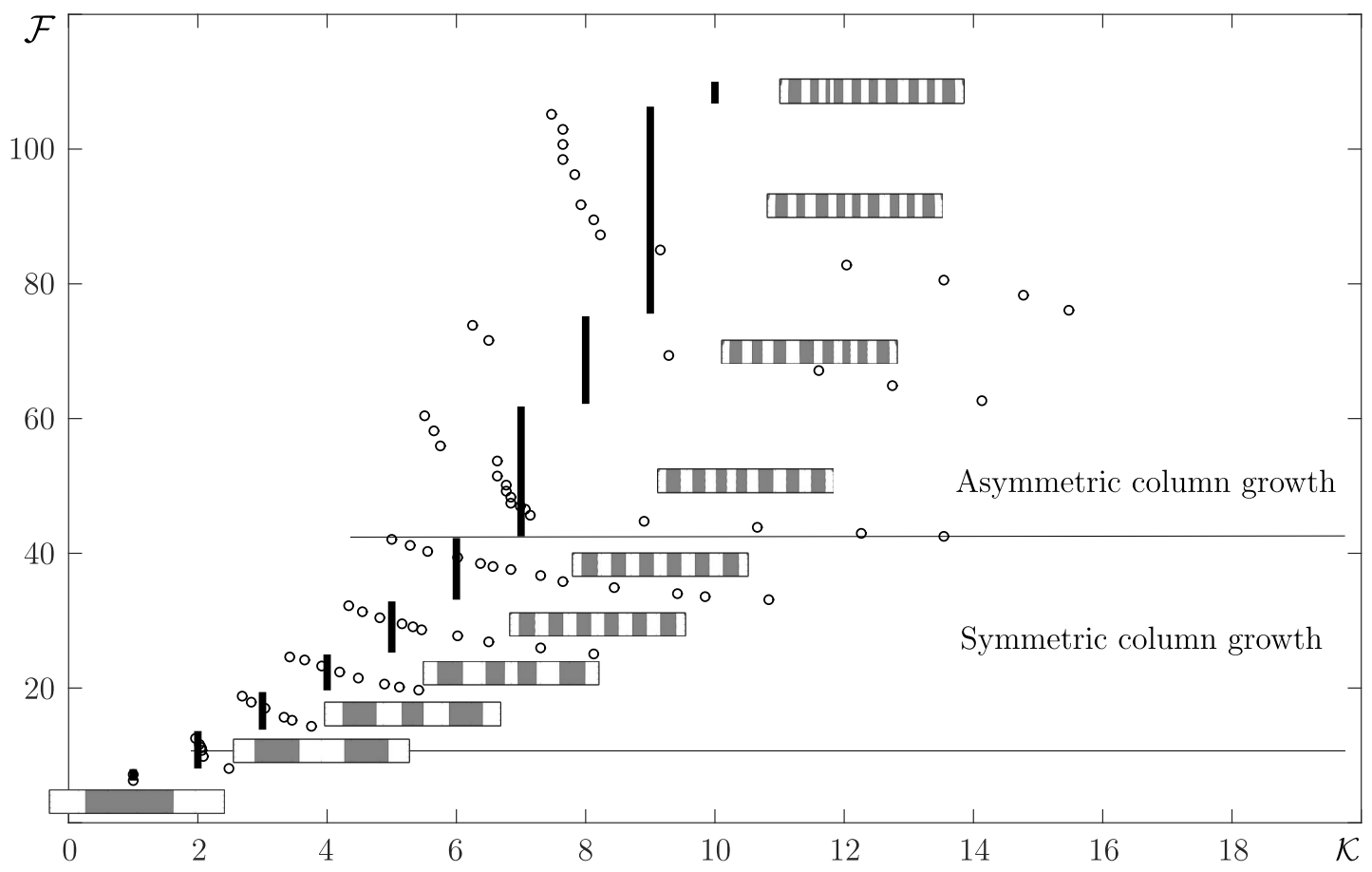

FiguRE 12. Diagram showing the relationship between vibrational forcing $\mathcal{F}$, the final average wavenumber $\langle\mathcal{K}\rangle$ (the number of column pairs; indicated by vertical lines) and the local wavenumber $\mathcal{K}$ (open dots) near the emerging column (defect). Horizontal lines mark the region of nearly symmetric central column growth; a qualitative transition to asymmetric defects occurs at $\mathcal{F} \simeq 42.8$. Simulations (see [134] for details) are performed with parameters appropriate to FC-40 and $20 \mathrm{cSt}$ silicone oil in a container with $L=60 \mathrm{~mm}$ and $2 H=7.5 \mathrm{~mm}$. The insets show selected final patterns.

Figure 12 shows results from a series of simulations in a larger aspect ratio container of length $L=60 \mathrm{~mm}$ and height $2 H=7.5 \mathrm{~mm}$. The dimensionless average wavenumber $\langle\mathcal{K}\rangle$ of each pattern, which is simply the number of column pairs measured across the midline, is shown (solid vertical lines) along with the local wavenumber $\mathcal{K}$ of the emerging defect (open circles). This local wavenumber (see Fig. 11) captures the growth of the new column from the point where its tip first crosses the midline to (for central defects) an extra wide column enclosing the next defect.

Note that the relationship between forcing and pattern wavenumber observed here is not that of equation (7.1), which gives $\mathcal{F} \propto \sqrt{k_{m}}$ for large enough $k_{m}$ (corresponding here to $\langle\mathcal{K}\rangle \gtrsim 3$ ). This suggests that, in addition to finite-size effects, wavenumber selection may be significantly affected by viscosity [99, 134], which favors the smaller wavenumbers observed.

\subsubsection{Strategies for control}

The main control parameter of the frozen wave instability is the applied velocity $v$ (equivalently, $\mathcal{F}$ ). Larger forcing amplitudes produces patterns of larger wavenumber. In microgravity, the final patterns take the form of columnar structures that can be quite regular at moderate forcing values not close to a mode transition, but grow more irregular and asymmetric for increasing forcing values. The mode transitions are characterized by the growth of a smaller column, or defect. With a diagram like that of Figure 12, a forcing amplitude can be chosen both to select the number of columns in the final state and the presence or not of irregularly sized columns (defects). 


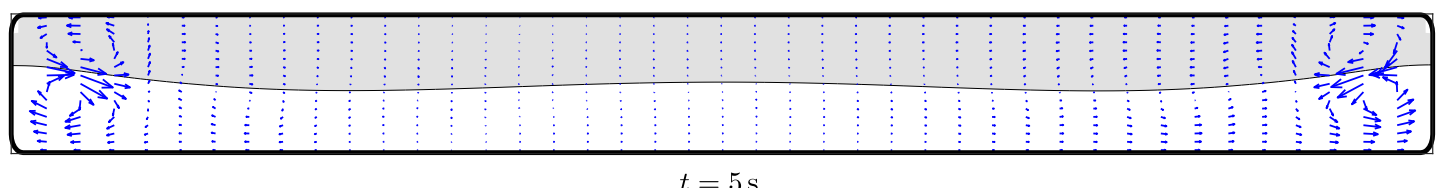

$$
t=5 \mathrm{~s}
$$

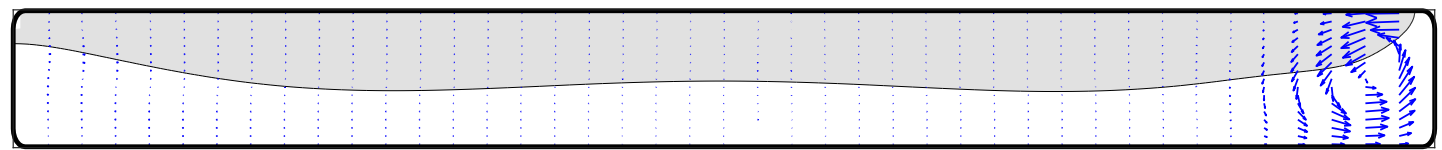

$t=10.325 \mathrm{~s}$

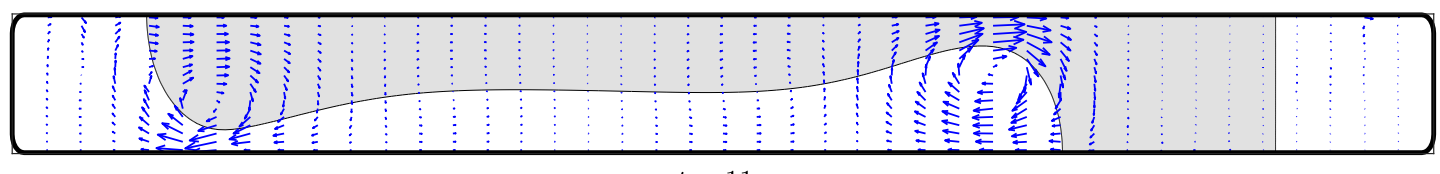

$t=11 \mathrm{~s}$

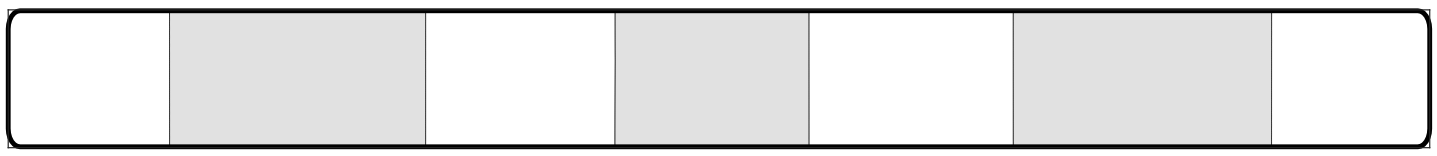

$t=12.5 \mathrm{~s}$

FIGURE 13. Evolution of the frozen wave instability (averaged over one excitation period) in weightlessness in a shallow rectangular container with $L=30 \mathrm{~mm}$ and $2 H=1.875 \mathrm{~mm}$ vibrated with $\mathcal{F} \simeq 7.59$. Parameters are appropriate to FC-40 and $20 \mathrm{cSt}$ silicone oil, with snapshots labeled by the time after initiation of the simulation (see [134] for details). The mean velocity field is shown in all but the final snapshot (scaling of arrow length is not constant between snapshots). Note the markedly asymmetric evolution of the interface, especially after the first (right) corner of the cell is covered by FC-40.

\subsection{Effect of the initial state}

The initial conditions of the fluid prior to the frozen wave instability are of crucial importance to the dynamics that follow. This includes, in particular, the size of the container and the extent to which the initial interface is flat and perpendicular to the forcing. Here we consider how these properties affect the final pattern.

The length $L$ of the container has an obvious effect on the pattern through wavenumber selection of the type illustrated in Figure 12. Since the final state must contain an integer number $n$ of column pairs (neglecting the complications of defects and irregularities), the average dimensional wavenumber satisfies $\langle k\rangle=2 \pi n / L$. The magnitude of the forcing determines the band of unstable modes, approximated by equation (7.1), while the length $L$ determines which of them are consistent with the lateral boundary conditions. Longer containers permit more modes and will exhibit more frequent mode transitions as the forcing is increased.

The height $2 H$ of the container has an important effect on the selected patterns as well. This is because the growth of longwave modes is affected earlier by interaction with the upper and lower boundaries. In shallow containers, the linear (low-amplitude) growth regime of a range of small $k$ modes is interrupted by collisions with these boundaries, which has the effect of breaking them into shorter columns and increasing the average wavenumber $\langle k\rangle$ of the final state. In deep containers, this interaction with the upper and lower boundaries is delayed, which provides more longwave modes with the chance to develop into columns. The added length of these columns, however, and their longer transient growth period, has the effect of amplifying initial nonuniformities and asymmetry.

The evolution of an immiscible interface in response to the frozen wave instability in a shallow container is illustrated in Figure 13. It can be seen that the initial perturbation (see the top panel at $t=5 \mathrm{~s}$ ) corresponds to $\langle\mathcal{K}\rangle=2$. This perturbation grows fastest near the endwalls and is slightly larger at the right compared to 

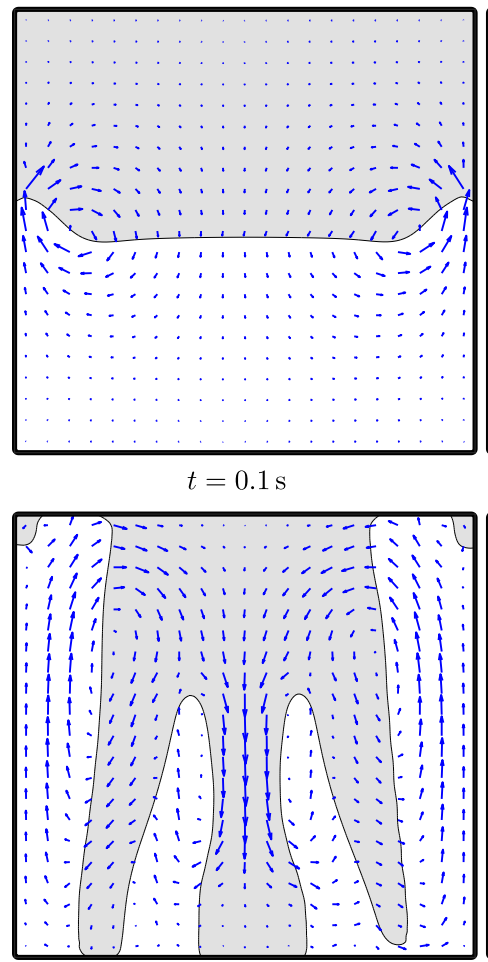

$t=0.6 \mathrm{~s}$

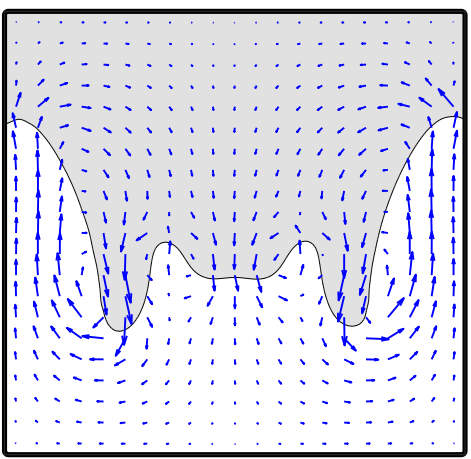

$t=0.325 \mathrm{~s}$

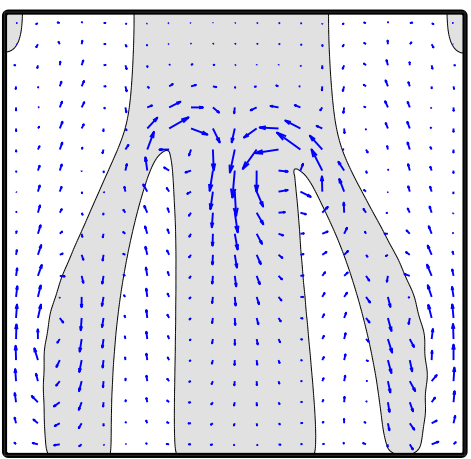

$t=0.8 \mathrm{~s}$

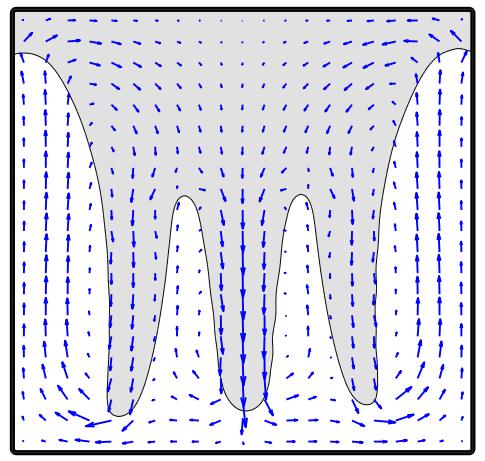

$t=0.45 \mathrm{~s}$

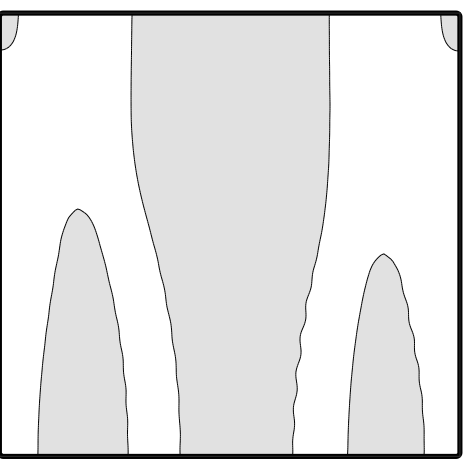

$t=1.4 \mathrm{~s}$

FiguRE 14. Evolution of the frozen wave instability in weightlessness in a deep container with $L=2 H=30 \mathrm{~mm}$ vibrated with $\mathcal{F} \simeq 37.97$. Parameters are appropriate to FC-40 and $20 \mathrm{cSt}$ silicone oil, with snapshots labeled by the time after initiation of the simulation (see [134] for details). The mean velocity field is shown by scaled arrows (scale not preserved between snapshots) for all but the final time. Asymmetric evolution associated with harmonic motion of the lateral column crests is observed.

the left, which is related to the harmonic waves at the two sides and their distinct phase in relationship to the initiation of the forcing $[134,168]$. After the frozen wave on the right side reaches the upper corner of the container, the dynamics of the interface are dominated by surface and contact forces acting to maintain the correct contact angle. This leads to rapid motion of the contact line (see the second panel at $t=10.325 \mathrm{~s}$ ) and the formation of a short column. A brief time later, the left side undergoes an analogous process. Successive collisions of this type dynamically increase the wavenumber of the columnar pattern so that the final state in this case (shown at $t=12.5 \mathrm{~s}$ ) has $\langle\mathcal{K}\rangle=3$. It is noteworthy, given the strongly nonlinear and asymmetric mechanism of wavenumber selection in such shallow containers, that the final structure is relatively symmetric.

The development of the frozen wave instability in the case of a deep container is illustrated in Figure 14. After initiation of the forcing, a perturbation with wavenumber close to $\langle\mathcal{K}\rangle=3$ is apparent. Again, the deformation of the interface is largest near the lateral walls. Compared to shallow containers, the frozen waves must achieve larger amplitude to reach the upper and lower boundaries. The increased transient time required for this amplifies the initial asymmetries, which are again largely due to the out-of-phase harmonic waves at each endwall. The final structure shows a significant degree of asymmetry, with several large defects resulting from the collision of the smaller columns (fingers) that are visible at $t=0.8 \mathrm{~s}$ with the larger lateral columns. This state also exhibits an asymmetric distribution of Faraday waves on the sides of the columns. Solutions of this type are indeed observed in microgravity experiments, both with immiscible liquids (see Fig. 10, also Salgado et al. [136]) and with miscible ones [99, 149]. 
In addition to the size of the container, the orientation of the initial interface with respect to the forcing axis has an important effect on the properties of the final state. Since the frozen wave instability depends on the imposition of an oscillatory shear flow, the forcing must have a significant component parallel to the initial interface. Deviations from this ideal configuration will occur, however, particularly in parabolic flight experiments where residual acceleration is not negligible and both its direction and magnitude vary [44].

Figure 15 shows how the inclination of the interface as the forcing begins can dramatically affect the final state. In the left column (a-d) of the figure, the initial interface is slightly inclined, with an angle of about $4.5^{\circ}$, and the frozen wave instability still develops much as it would for a perfectly horizontal interface. The final state consists of approximately four pairs of columns, similar to the results of the simulations shown in Figures 11-14. However, with the initial interface inclined at about $27^{\circ}$, as in the right column (e-h), the frozen wave instability does not develop in a clear manner at all. While two lateral perturbations do appear to grow [as in (f)], the interior columns are suppressed or subsumed by those. The overall effect is to rotate the average interface into a nearly vertical position and the final state is approximated by a single column pair, with the heavier fluid primarily located on the left and the lighter one on the right.

The behaviour of the vibrated interface with initial inclination can be understood as kind of competition between the frozen wave instability and the vibroequilibria effect. If the inclination is small enough, oscillatory shear flow prevails and the frozen wave instability is triggered. If the inclination is large enough, the shear flow is suppressed and the vibroequilibria effect acts to rotate the interface into a nearly perpendicular orientation before recognizable frozen waves can develop. Intermediate angles lead to varied and complex patterns, often with smaller auxiliary columns or fingers; see [133] for more details.

Figure 16 shows the results of a series of numerical simulations with parameters appropriate to FC-40 and $20 \mathrm{cSt}$ silicone oil, where the initial inclination of the interface was varied by small steps. While the final states can be complicated, with columns of irregular width and secondary Faraday waves, the general effect of increasing inclination is to drive a transition from nearly regular columns of higher wavenumber $(\langle\mathcal{K}\rangle=3)$ to more asymmetric patterns of fewer columns and, finally, a single column pair state $(\langle\mathcal{K}\rangle=1)$ with an almost complete lateral separation of the two fluids.

The vibroequilibria effect undoubtedly plays an important role when the initial interface is inclined, leading to further rotation and a corresponding suppression of the shear flow that drives the frozen wave instability. Even in the ideal case of a flat horizontal initial interface, however, the vibroequilibria effect has a significant impact on the frozen wave instability. This is because the effect is most pronounced near the lateral walls where the inhomogeneities of the velocity field are strongest. The heavier fluid is driven to climb these lateral walls (see Fig. 9). While frozen waves excited on an infinitely long flat interface may take any position (phase) in $x$, this is not so in finite containers. The fact that the vibroequilibria effect pushes the heavier fluid upward along the sides means that frozen waves compatible with this are selected over those that are not (i.e., with a column of lighter fluid along one or both of the sides). Indeed, in all the simulations of Figures 11-14, the heavier fluid always ends up along the lateral walls (see also [60, 96, 99, 134, 168]). This symmetry-breaking effect of vibroequilibria on the frozen wave instability is investigated in [168]. Furthermore, in promoting the same behavior at both lateral walls, the vibroequilibria effect is likely responsible, at least in part, for the high degree of left-right reflection symmetry in the patterns obtained with no average initial inclination over a wide range of forcing amplitudes (see Fig. 12).

\subsubsection{Strategies for control}

While the amplitude of the forcing is the principal means of controlling the wavenumber of frozen wave patterns in microgravity, the size of the container plays a crucial role as well. The length $L$ determines which mode numbers are allowed, while the height $2 H$ has a significant influence on their nonlinear growth. Shallow containers interrupt the growth of longwave modes through repeated collisions with the upper and lower boundaries, yielding final columnar states with a higher wavenumber than that of the fastest growing perturbation. Deep containers, associated with longer transient times and longer columns, amplify preexisting asymmetries and lead to more irregular columnar structures. The features of the pattern can, therefore, be manipulated 


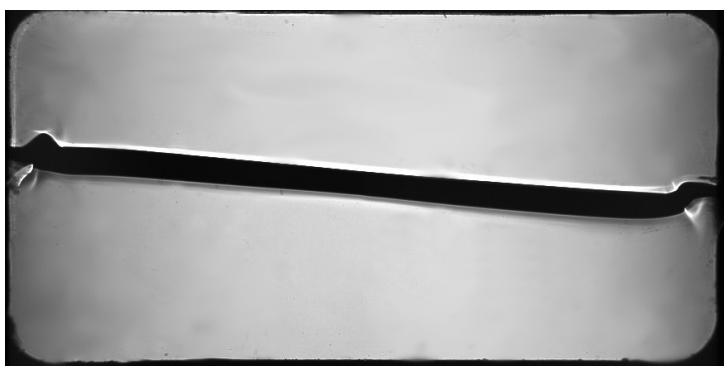

(a) $t=0 \mathrm{~s}$

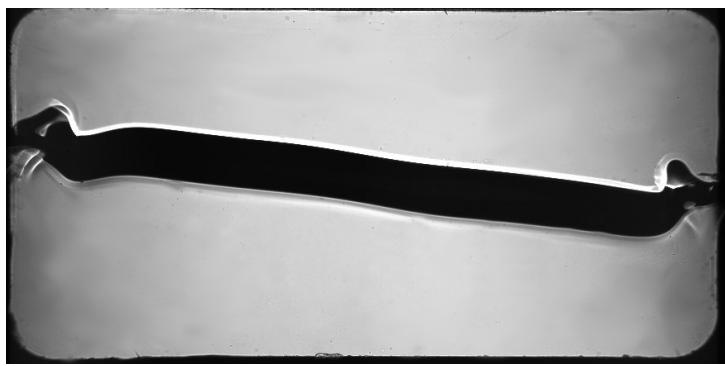

(b) $t=0.828 \mathrm{~s}$

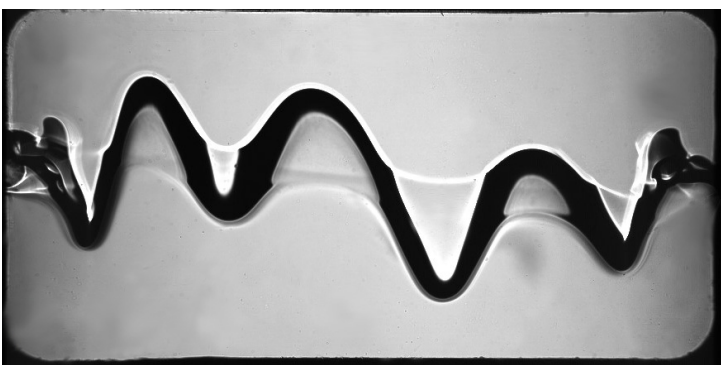

(c) $t=2.281 \mathrm{~s}$

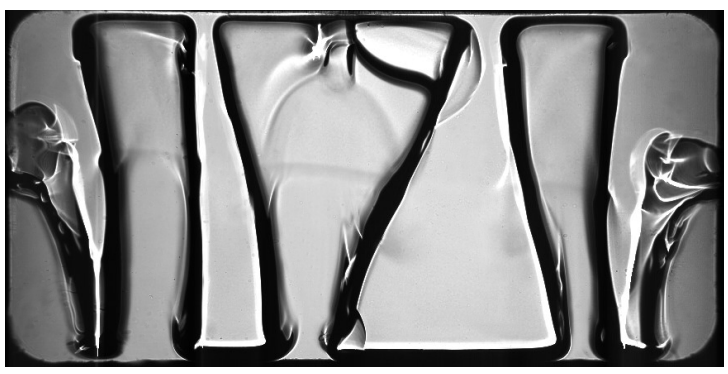

(d) $t=6.984 \mathrm{~s}$

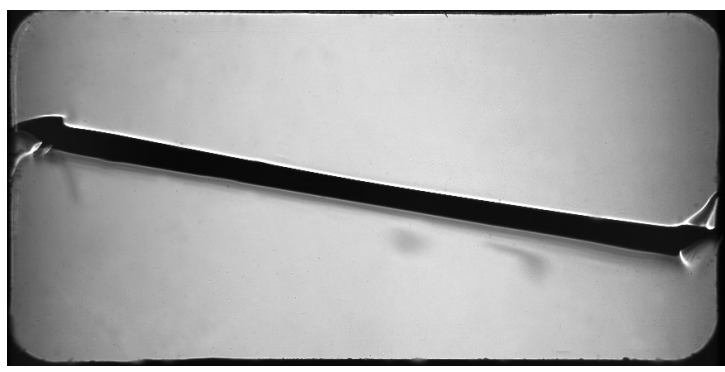

(e) $t=0 \mathrm{~s}$

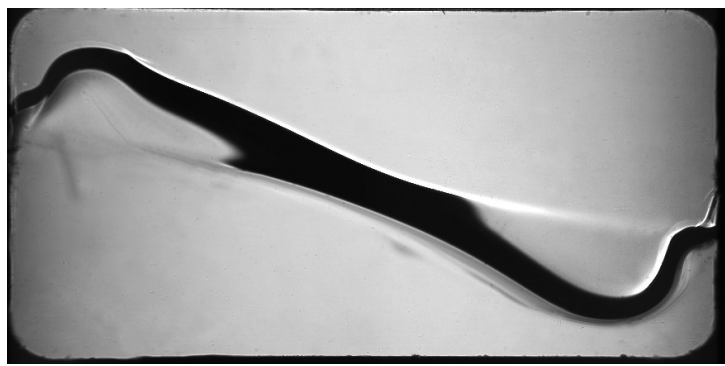

(f) $t=0.828 \mathrm{~s}$

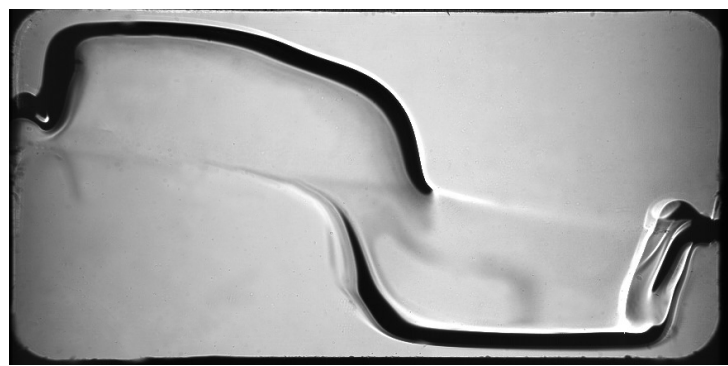

(g) $t=4.172 \mathrm{~s}$

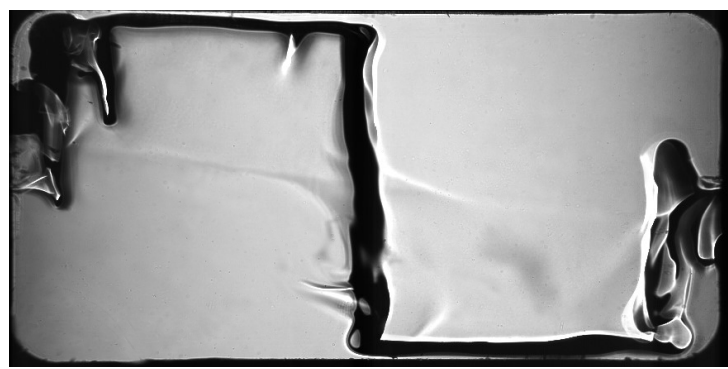

(h) $t=5.328 \mathrm{~s}$

FiguRE 15. Effect of initial interface orientation on the development of the frozen wave instability in microgravity in a container with $L=15 \mathrm{~mm}$ and $2 H=7.5 \mathrm{~mm}$. The layers are miscible water-isopropanol mixtures with water mass fractions of 0.9 and 0.5 and densities of $\rho_{1}=980.9 \mathrm{~kg} / \mathrm{m}^{3}$ and $\rho_{2}=902.4 \mathrm{~kg} / \mathrm{m}^{3}$, respectively. The forcing is $v=219.9 \mathrm{~mm} / \mathrm{s}$ in (a-d) and $v=219.4 \mathrm{~mm} / \mathrm{s}$ in $(\mathrm{e}-\mathrm{h})$ while the initial inclination increases from approximately $4.5^{\circ}$ in (a-d) to $27^{\circ}$ in (e-h) [as measured in (f)]. Apparent breaks in the interface [as in (g)] are optical effects. More details can be found in [133]. 
(a) $\mathcal{F}=28.48$

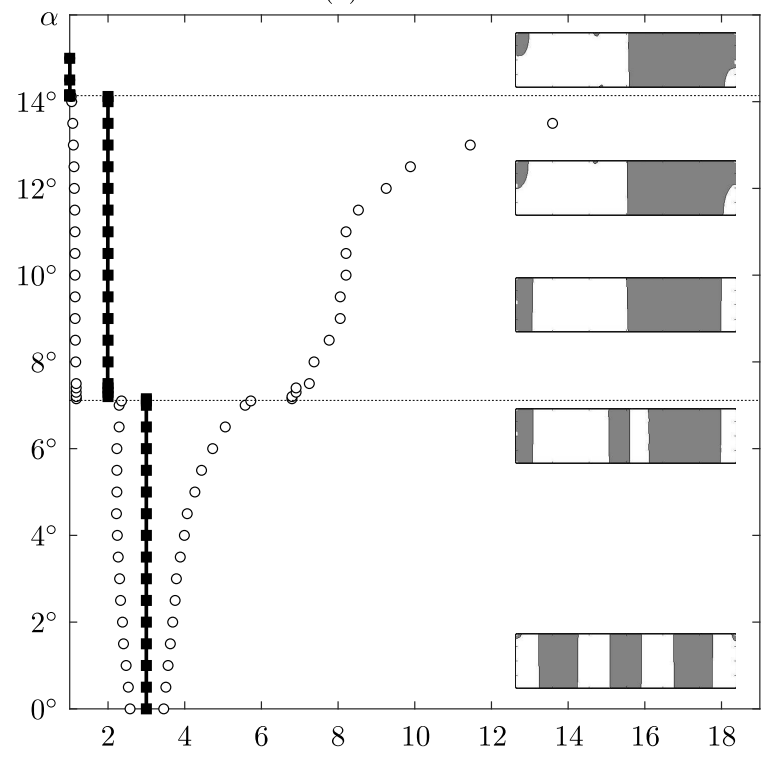

(b) $\mathcal{F}=37.97$

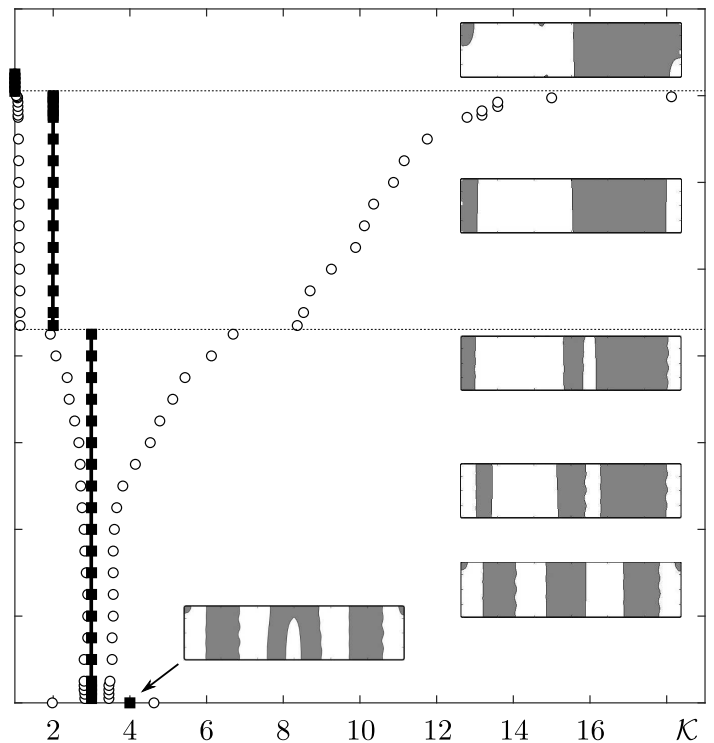

FiguRE 16. Diagrams showing the effect of initial interface angle $\alpha$ (in degrees) on the dimensionless pattern wavenumber measured at the midplane for two applied vibrational velocities: (a) $\mathcal{F}=28.48$, (b) $\mathcal{F}=37.97$. Simulations (see [133] for details) are performed with parameters appropriate to FC-40 and $20 \mathrm{cSt}$ silicone oil in a domain with $L=30 \mathrm{~mm}$ and $2 H=7.5 \mathrm{~mm}$. The average wavenumber $\langle\mathcal{K}\rangle$ is indicated by solid black squares, while the minimum and maximum local wavenumbers are shown with open circles. Insets show selected final patterns.

through appropriate choice of container size. In particular, if regular columns are desired, both shallow and deep containers should be avoided, while the length should permit a moderate number of column pairs at the given forcing value; for large mode numbers, the columns become more asymmetric (see Fig. 12).

It is also clear that the initial inclination of the surface is a critical parameter. Only relatively small inclinations permit the regular oscillatory shear flow that generates frozen waves. If the inclination is more than a few degrees (see Fig. 16), the final columnar states are quite asymmetric, with some columns much wider than others. With still more inclination, the frozen wave instability is effectively suppressed and the final state is similar to a single column pair with the two fluids largely separated at opposite sides, which can be understood as a consequence of the vibroequilibria effect. If regular columns are desired, the initial inclination must be controlled with sufficient accuracy. If left-right separation is the goal, this can be achieved with a relatively small angle (on the order of $10^{\circ}$ for the parameters of Fig. 16) between the forcing axis and the average initial surface profile.

\subsection{Nonlinear effects}

The development of frozen waves in microgravity is inherently nonlinear due to their rapid growth into large columnar structures. There is, in this sense, less distinction than in other fluid systems between factors that influence the linear problem and those that affect nonlinear interactions. Several of the factors that influence frozen wave development, including container length and height, the inclination of the initial interface, and the vibroequilibria effect have been discussed above. In particular, the effect of container height is largely nonlinear, either through collisions with the upper and lower boundaries in shallow containers, or through the growth and interaction of long columns and fingers in deep containers.

Here, we look at the nonuniform and nonlinear way in which the frozen wave patterns develop in finite containers, often moving from the outside in, as illustrated in Figures 11, 13 and 14. This means that interior waves (columns) grow between a larger column on one side and a more quiescent region on the other. It can 
be expected that the adjacent larger column helps select the wavenumber of the growing column and that this interaction dominates over interactions with more distant columns, like those developing inward from the opposite side. This strong nonlinear interaction means that defects, if they occur, are likely to appear in the center of the container where the inward moving column formation sequences from the left and right meet. Such defects can be understood as a type of wavenumber frustration that is concentrated in the central region because the frozen waves there are the last to develop into large columns.

While reasons for this nonuniform growth can be found in the inhomogeneous velocity field near the lateral boundaries and in the associated vibroequilibria effect, it appears that the manner in which the forcing is initiated is also important. If, as in the simulations described here (where it is initiated smoothly over $0.25 \mathrm{~s}$; see [136]), the forcing is turned on rapidly, then the agitation of the fluid is concentrated at first near the lateral walls, where harmonic waves form and the vibroequilibria effect leads to upward motion of the contact lines. The net result of this is to provide a finite perturbation that gives an advantage to the frozen waves (columns) beginning to grow in those lateral regions. As they grow, they induce neighboring columns to grow, initiating a process that generates successive columns from the outside in.

This type of column formation process holds over a substantial range of forcing values but the influence of the lateral walls diminishes as the wavenumber increases and, at some point, defects begin to appear at off-center locations. This transition, despite being associated with a highly nonlinear process, occurs with a regularity that is suggestive of spontaneous symmetry breaking, as described in Troitiño et al. [168]. Figure 17 illustrates this symmetry-breaking transition with increasing forcing in a container of length $L=60 \mathrm{~mm}$ and height $2 H=7.5 \mathrm{~mm}$. The left-right asymmetry of those solutions is characterized by the asymmetry function

$$
\mathcal{A}=\frac{100}{L H} \int_{-H}^{H} \int_{0}^{L / 2}|\phi(x, y)-\phi(-x, y)| \mathrm{d} x \mathrm{~d} y,
$$

where $\phi$ is a level-set function that takes the value 0 in one fluid and 1 in the other (see [168] for details).

\subsubsection{Strategies for control}

Since the frozen wave instability in microgravity leads to large columnar patterns, nonlinear interactions are an essential aspect of the system. Aside from interactions with the boundaries of the container, we have seen that the spatially inhomogeneous nature of the forcing is important. When the forcing is begun rapidly, the disturbances near the lateral walls, both due to harmonic surface waves and to the vibroequilibria effect, act to initiate the growth of perturbations where the heavier fluid moves upward along these walls. The development of frozen waves into columns then proceeds from the outside in, with significant nonlinear interaction. The chains of column formation moving inward from the left and right meet in the central region, where defects are most likely to occur. This type of development is conducive to (approximately) left-right symmetric patterns and occurs, as mentioned above, for forcing values corresponding to a moderate number of column pairs. If the aspect ratio is increased, or the forcing amplitude raised, so that the average wavenumber $\langle\mathcal{K}\rangle$ becomes large, the influence of the lateral walls diminishes and defects begin to occur at off-center locations, leading to more asymmetric patterns [168]. The manner in which the forcing is begun and the lateral boundary conditions thus appear to play an important part in selecting the final state, with the corresponding potential for controlling it.

\section{CONCLUSiOn}

This review considered several classical fluid instabilities driven by gravity, surface tension, thermal energy, and kinetic energy (applied accelerations or relative motion). Some of the methods used to control these instabilities were discussed, including strategies based on the choice of initial conditions, such as fluid properties and container size, adjustment of forcing function characteristics, such as amplitude and frequency content, the inclusion of additional forcing or feedback, and the identification of parameters that affect nonlinear growth and associated pattern selection processes. 

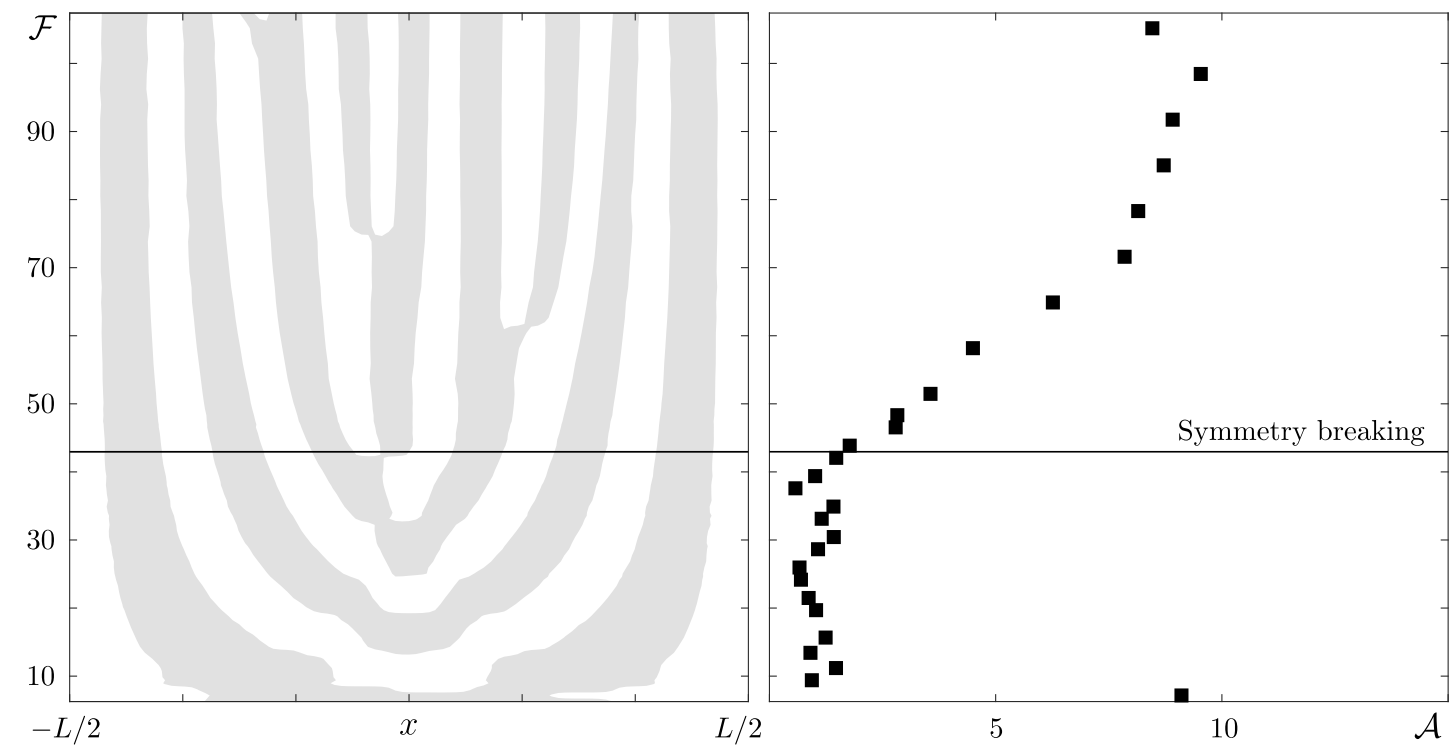

Figure 17. (a) Density distribution of the final steady state (lighter liquid shaded) along the horizontal midline (i.e., the location of the initial unperturbed surface) as a function of $\mathcal{F}$ for a container with $L=60 \mathrm{~mm}$ and $2 H=7.5 \mathrm{~mm}$. (b) The asymmetry $\mathcal{A}$ [see Eq. (7.4)] of these same solutions. The column growth and defect locations become asymmetric at $\mathcal{F} \simeq 42.8$ (marked by the horizontal line), as reflected in the increase of $\mathcal{A}$. Simulations (see [168] for details) are performed with parameters appropriate to FC-40 and $20 \mathrm{cSt}$ silicone oil.

Gravity was seen to play a crucial role in nearly all of these fluid instabilities, both in setting the threshold condition and as a restoring force to limit the growth of unstable perturbations. The absence of strong gravity, as in microgravity environments, means that many fluid instabilities develop in a decidedly different fashion than they do in normal gravity. In general, fluids are much less constrained and may readily adjust their position (center of mass) and shape in response to the forces they encounter. This difference both explains the appearance of novel behavior and provides an intriguing opportunity for its manipulation and control.

The specific case of the frozen wave instability in microgravity was used to illustrate these ideas with a summary of recent experimental and numerical results. Frozen waves develop in a much different manner without the restoring force of gravity. This is anticipated by the vanishing of the instability threshold as $g \rightarrow 0$ in the infinitely extended, inviscid limit so that, for finite forcing amplitude, there is always a band of unstable modes. Furthermore, without gravity, these modes grow very rapidly, with no stabilizing cubic nonlinearity, and develop into large columnar structures spanning the full height of the container.

Several salient factors were discussed for their effect on the final columnar states including the forcing amplitude, the container size, the average angle between the initial surface and the forcing axis, the vibroequilibria effect, and the inhomogeneous, nonlinear way in which the columns grow, developing first near the lateral walls and moving successively inward. A judicious choice of the relevant parameters allows one to control the frozen wave instability to a substantial degree, including the number, regularity and left-right symmetry of the final columnar states. Uniform columns or irregular, asymmetric patterns can be selected, for example, as well as an almost complete lateral separation of the two fluids.

Acknowledgements. The work of J.P. and P.S.S. was supported by the Ministerio de Economía y Competitividad under Project No. ESP2015-70458-P. The work of V.S. and V.Y. was supported by the PRODEX programme of the Belgian Federal Science Policy Office. 


\section{REFERENCES}

[1] G. Ahlers, F.F. Araujo, D. Funfschilling, S. Grossmann and D. Lohse, Non-Oberbeck-Boussinesq Effects in Gaseous RayleighBénard Convection. Phys. Rev. Lett. 98 (2007) 054501.

[2] A.V. Anilkumar, R.N. Grugel, X.F. Shen, C.P. Lee and T.G. Wang, Control of thermocapillary convection in a liquid bridge by vibration. J. Appl. Phys. 73 (1993) 4165-4170.

[3] H. Arbell and J. Fineberg, Pattern formation in two-frequency forced parametric waves. Phys. Rev. E 65 (2002) 036224.

[4] P. Ashwin and A. Zaikin, Pattern selection: the importance of "how you get there". Biophys. J. 108 (2015) $1307-1308$.

[5] H. Ayanle, A.J. Bernoff and S. Lichter. Spanwise modal competition in cross-waves. Physica D 43 (1990) 87-104.

[6] T. Azami, S. Nakamura and T. Hibiya, Effect of oxygen on thermocapillary convection in a molten silicon column under microgravity. J. Electrochem. Soc. 148 (2001) G185.

[7] L. Bárcena, J. Shiomi and G. Amberg. Control of oscillatory thermocapillary convection with local heating. J. Cryst. Growth 286 (2006) 502-511.

[8] B.J.S. Barnard and W.G. Pritchard, Cross-waves. Part 2. Experiments. J. Fluid Mech. 55 (1972) 245-255.

[9] O.A. Basaran, H. Gao and P.P. Bhat. Nonstandard inkjets. Annu. Rev. Fluid Mech. 45 (2013) 85-113.

[10] J.M. Becker and J.W. Miles. Standing radial cross-waves. J. Fluid Mech. 222 (1991) 471-499.

[11] G. Beintema, A. Corbetta, L. Biferale and F. Toschi. Controlling Rayleigh-Bénard convection via reinforcement learning. J. Turbul. (2020) 1-21.

[12] R. Bellman and R.H. Pennington, Effects of surface tension and viscosity on Taylor instability. Quart. Appl. Math. 12 (1954) $151-162$.

[13] H. Bénard, Les tourbillons cellulaires dans une nappe liquide. Rev. Gén. Sciences Pure Appl. 11 (1900) 1261-1271, 1309-1328.

[14] T.B. Benjamin and F. Ursell. The stability of a plane free surface of a liquid in vertical periodic motion. Proc. Roy. Soc. Lond. A 225 (1954) 505-515.

[15] J. Berg and A. Acrivos. The effect of surface active agents on convection cells induced by surface tension. Chem. Eng. Sci. 20 (1965) 737-745.

[16] K. Beyer, I. Gawriljuk, M. Günther, I. Lukovsky and A. Timokha. Compressible potential flows with free boundaries. Part I: Vibrocapillary equilibria. Z. Angew. Math. Mech. 81 (2001) 261-271.

[17] K. Beyer, M. Günther and A. Timokha, Variational and finite element analysis of vibroequilibria. Comput. Methods Appl. Math. 4 (2004) 290-323.

[18] D. Beysens, Vibrations in space as an artificial gravity? Europhysics News 37 (2006) 22-25.

[19] N.K. Bezdenezhnykh, V.A. Briskman, D.V. Lyubimov, A.A. Cherepanov and M.T. Sharov. Control of stability of a fluid interface by means of vibrations, electric and magnetic fields, In Third All-Union Seminar on Hydromechanics and Heat and Mass Transfer in Zero Gravity, Abstracts of Papers (in Russian). (1984) 18-20.

[20] T. Bickel, Effect of surface-active contaminants on radial thermocapillary flows. Eur. Phys. J. E. 42 (2019) 131.

[21] E. Bodenschatz, W. Pesch and G. Ahlers, Recent developments in Rayleigh-Bénard convection. Annu. Rev. Fluid Mech. 32 (2000) 709-778.

[22] J.C. Brice, Crystal growth. Blackie and Son (1986).

[23] A. Burkert and D.N.C. Lin, Thermal instability and the formation of clumpy gas clouds. Astrophys. J. 537 (2000) $270-282$.

[24] V. Bychkov, M. Modestov, V. Akkerman and L.-E. Eriksson, The Rayleigh-Taylor instability in inertial fusion, astrophysical plasma and flames. Plasma Phys. Controlled Fusion 49 (2007) B513-B520.

[25] R.V. Cakmur, D.A. Egolf, B.B. Plapp and E. Bodenschatz. Bistability and competition of spatiotemporal chaotic and fixed point attractors in Rayleigh-Bénard convection. Phys. Rev. Lett. 79 (1997) 1853-1856.

[26] J.R. Carpenter, E.W. Tedford, M. Rahmani and G.A. Lawrence, Holmboe wave fields in simulation and experiment. J. Fluid Mech. 648 (2010) 205-223.

[27] J.K. Castelino, D.J. Ratliff, A.M. Rucklidge, P. Subramanian and C.M. Topaz. Spatiotemporal chaos and quasipatterns in coupled reaction-diffusion systems. Physica D 409 (2020) 132475.

[28] Y.-J. Chen, R. Abbaschian and P.H. Steen. Thermocapillary suppression of the Plateau-Rayleigh instability: a model for long encapsulated liquid zones. J. Fluid Mech. 485 (2003) 97-113.

[29] C.-H. Chun and W. Wuest, Experiments on the transition from the steady to the oscillatory Marangoni-convection of a floating zone under reduced gravity effect. Acta Astronaut. 6 (1979) 1073-1082.

[30] I. Cisse, G. Bardan and A. Mojtabi, Rayleigh Bénard convective instability of a fluid under high-frequency vibration. Int. J. Heat Mass Transf. 47 (2004) 4101-4112.

[31] S.H. Davis. The stability of time-periodic flows. Annu. Rev. Fluid Mech., 8 (1976) 57-74.

[32] Y. Ding and P. Umbanhowar, Enhanced Faraday pattern stability with three-frequency driving. Phys. Rev. E 73 (2006) 046305.

[33] S. Douady, Experimental study of the Faraday instability. J. Fluid Mech. 221 (1990) 383-409.

[34] P. Drazin, Dynamical Meteorology | Kelvin-Helmholtz Instability, In G.R. North, J. Pyle and F. Zhang, editors, Encyclopedia of Atmospheric Sciences. Academic Press, Oxford, second edition (2015) 343-346.

[35] R. Dressler and N. Sivakumaran. Non-contaminating method to reduce Marangoni convection in microgravity float zones. J. Cryst. Growth 88 (1988) 148-158.

[36] T. Driessen, P. Sleutel, J. Dijksman, R. Jeurissen and D. Lohse. Control of jet breakup by a superposition of two RayleighPlateau-unstable modes. J. Fluid Mech. 749 (2014) 275-296. 
[37] V. Duclaux, C. Clanet and D. Quéré, The effects of gravity on the capillary instability in tubes. J. Fluid Mech. 556 (2006) 217-226.

[38] W.S. Edwards and S. Fauve. Parametrically excited quasicrystalline surface waves. Phys. Rev. E 47 (1993) R788-R791.

[39] W.S. Edwards and S. Fauve, Patterns and quasi-patterns in the Faraday experiment. J. Fluid Mech. 278 (1994) $123-148$.

[40] J.M. Ezquerro, A. Bello, P. Salgado Sanchez, A. Laveron-Simavilla and V. Lapuerta, The Thermocapillary Effects in Phase Change Materials in Microgravity experiment: Design, preparation and execution of a parabolic flight experiment. Acta Astronaut. 162 (2019) 185-196.

[41] J.M. Ezquerro, P. Salgado Sanchez, A. Bello, J. Rodriguez, V. Lapuerta and A. Laveron-Simavilla, Experimental evidence of thermocapillarity in phase change materials in microgravity: measuring the effect of Marangoni convection in solid/liquid phase transitions. Int. Commun. Heat Mass Transf. 113 (2020) 104529.

[42] O. Faltinsen and A. Timokha. Sloshing. Cambridge Univ. Press (2009).

[43] M. Faraday, On a peculiar class of acoustical figures; and on certain forms assumed by groups of particles upon vibrating elastic surfaces. Phil. Trans. R. Soc. Lond. 121 (1831) 299-340.

[44] J. Fernandez, P. Salgado Sánchez, I. Tinao, J. Porter and J.M. Ezquerro, The CFVib experiment: control of fluids in microgravity with vibrations. Microgravity Sci. Technol. 29 (2017) 351-364.

[45] J. Fernández, I. Tinao, J. Porter and A. Laverón-Simavilla, Instabilities of vibroequilibria in rectangular containers. Phys. Fluids 29 (2017) 024108.

[46] G.B. Field, Thermal instability. Astrophys. J. 142 (1965) 531-567.

[47] J. Fröhlich, P. Laure and R. Peyret, Large departures from Boussinesq approximation in the Rayleigh-Bénard problem. Phys. Fluids A 4 (1992) 1355-1372.

[48] T. Funada and D.D. Joseph, Viscous potential flow analysis of Kelvin-Helmholtz instability in a channel. J. Fluid Mech. 445 (2001) 263-283.

[49] G. Gandikota, D. Chatain, S. Amiroudine, T. Lyubimova and D. Beysens, Frozen-wave instability in near-critical hydrogen subjected to horizontal vibration under various gravity fields. Phys. Rev. E 89(2014) 012309.

[50] R.F. Ganiev, V.D. Lakiza and A.S. Tsapenko, Dynamic behavior of the free liquid surface subject to vibrations under conditions of near-zero gravity. Sov. Appl. Mech. 13 (1977) 499-503.

[51] Y. Gaponenko, A. Mialdun and V. Shevtsova, Pattern selection in miscible liquids under periodic excitation in microgravity: Effect of interface width. Phys. Fluids 30 (2018) 062103.

[52] Y. Gaponenko, V. Yasnou, A. Mialdun, A. Nepomnyashchy and V. Shevtsova, Hydrothermal waves in a liquid bridge subjected to a gas stream along the interface. J. Fluid Mech. 908 (2021) A34.

[53] Y.A. Gaponenko, M.M. Torregrosa, V. Yasnou, A. Mialdun and V. Shevtsova, Interfacial pattern selection in miscible liquids under vibration. Soft Matter 11 (2015) 8221-8224.

[54] Y. Garrabos, D. Beysens, C. Lecoutre, A. Dejoan, V. Polezhaev and V. Emelianov, Thermoconvectional phenomena induced by vibrations in supercritical $\mathrm{SF}_{6}$ under weightlessness. Phys. Rev. E $\mathbf{7 5}$ (2007) 056317.

[55] C.J.R. Garrett, On Cross-waves. J. Fluid Mech. 41 (1970) 837-849.

[56] I. Gavrilyuk, I. Lukovsky and A. Timokha, Two-dimensional variational vibroequilibria and Faraday'sdrops. Z. Angew. Math. Phys. 55 (2004) 1015-1033.

[57] A. Gelfgat, P. Bar-Yoseph and A. Solan, Effect of axial magnetic field on three-dimensional instability of natural convection in a vertical Bridgman growth configuration. J. Cryst. Growth 230 (2001) 63-72.

[58] G.Z. Gershuni and E.M. Zhukhovitskii, Free thermal convection in a vibrational field under conditions of weightlessness. Sov. Phys. Dokl. 24 (1979) 894-896.

[59] A.V. Getling, Rayleigh-Bénard convection, World Scientific (1998).

[60] D. Gligor, P. Salgado Sánchez, J. Porter and V. Shevtsova, Influence of gravity on the frozen wave instability in immiscible liquids. Phys. Rev. Fluids 5 (Aug 2020) 084001.

[61] P.M. Gresho and R.L. Sani, The effects of gravity modulation on the stability of a heated fluid layer. J. Fluid Mech. 40 (1970) 783-806.

[62] S. Haefner, M. Benzaquen, O. Bäumchen, T. Salez, R. Peters, J.D. McGraw, K. Jacobs, E. Raphaël and K. Dalnoki-Veress, Influence of slip on the Plateau-Rayleigh instability on a fibre. Nat. Commun. 6 (2015) 7409.

[63] T. Havelock, LIX. Forced surface-waves on water. Phil. Mag. 8 (1929) 569-576.

[64] M. Haynes, E. Vega, M. Herrada, E. Benilov and J. Montanero, Stabilization of axisymmetric liquid bridges through vibrationinduced pressure fields. J. Colloid Interface Sci. 513 (2018) 409-417.

[65] H. Helmholtz, On discontinuous movements of fluids. Philos. Mag. 36 (1868) 337-346.

[66] J. Holmboe, On the behavior of symmetric waves in stratified shear layers. Geofys. Publ. 24 (1962) 67-113.

[67] L.E. Howle, Active control of Rayleigh-Bénard convection. Phys. Fluids 9 (1997) 1861-1863.

[68] I. Mutabazi, J. E. Wesfreid and E. Guyon, editors, Dynamics of spatio-temporal cellular structures. Vol. 207 of Springer Tracts in Modern Physics, Springer-Verlag, New York (2006).

[69] S.V. Jalikop and A. Juel, Steep capillary-gravity waves in oscillatory shear-driven flows. J. Fluid Mech. 640 (2009) 131-150.

[70] D.L. Jassby, Evolution and Large-Electric-Field Suppression of the Transverse Kelvin-Helmholtz Instability. Phys. Rev. Lett. 25 (1970) 1567-1570.

[71] A.F. Jones, The generation of cross-waves in a long deep channel by parametric resonance. J. Fluid Mech. 138 (1984) 53-74.

[72] B.L. Jones, P.H. Heins, E.C. Kerrigan, J.F. Morrison and A.S. Sharma, Modelling for robust feedback control of fluid flows. J. Fluid Mech. 769 (2015) 687-722. 
[73] R. Jurado, J. Pallarés, J. Gavaldà and X. Ruiz, Effect of reboosting manoeuvres on the determination of the Soret coefficients of DCMIX ternary systems. Int. J. Therm. Sci. 142 (2019) 205-219.

[74] M. Jurisch and W. Löser, Analysis of periodic non-rotational W striations in Mo single crystals due to nonsteady thermocapillary convection. J. Cryst. Growth 102 (1990) 214-222.

[75] P.L. Kapitza, Dynamic stability of a pendulum when its point of suspension vibrates. Soviet Phys. JETP. 21 (1951) 588-597.

[76] R.E. Kelly, Stabilization of Rayleigh-Bénard convection by means of a slow nonplanar oscillatory flow. Phys. Fluids A 4: (1992) 647-648.

[77] L. Kelvin, Mathematical and physical papers, IV, hydrodynamics and general dynamics. Cambridge Univ. Press (1910).

[78] A. Khait and L. Shemer. Nonlinear wave generation by a wavemaker in deep to intermediate water depth. Ocean Eng. 182 (2019) 222-234.

[79] A. Kidess, S. Kenjereš and C.R. Kleijn, The influence of surfactants on thermocapillary flow instabilities in low Prandtl melting pools. Phys. Fluids 28 (2016) 062106.

[80] T.S. Krasnopolskaya and G.J.F.V. Heijst, Wave pattern formation in a fluid annulus with a radially vibrating inner cylinder. J. Fluid Mech. 328 (1996) 229-252.

[81] M. Kudo, Y. Akiyama, S. Takei, K. Motegi and I. Ueno, Effect of ambient air flow on thermocapillary convection in a full-zone liquid bridge. Interfacial Phenom. Heat Transf. 3 (2015) 231-242.

[82] A. Kudrolli and J.P. Gollub, Patterns and spatio-temporal chaos in parametrically forced surface waves: a systematic survey at large aspect ratio. Physica D 97 (1996) 133-154.

[83] A. Kudrolli, B. Pier and J.P. Gollub, Superlattice patterns in surface waves. Physica D 123 (1998) 99-111.

[84] K. Kumar, Linear theory of Faraday instability in viscous liquids. Proc. R. Soc. A 452 (1996) 1113-1126.

[85] K. Kumar and L.S. Tuckerman, Parametric instability of the interface between two fluids. J. Fluid Mech. 279 (1994) 49-68.

[86] L.D. Landau and E.M. Lifshitz, Fluid mechanics. Vol. 6 of Course of Theoretical Physics. Pergamon Books Ltd., second edition, (1987).

[87] M. Lappa. Review: Possible strategies for the control and stabilization of Marangoni flow in laterally heated floating zones. Fluid Dyn. Mater. Process. 1 (2005) 171-188.

[88] J. Lindl, Development of the indirect-drive approach to inertial confinement fusion and the target physics basis for ignition and gain. Phys. Plasmas 2 (1995) 3933-4024.

[89] B.J. Lowry and P.H. Steen, Capillary surfaces: stability from families of equilibria with application to the liquid bridge. Proc. R. Soc. A 449 (1995) 411-439.

[90] B.J. Lowry and P.H. Steen, Flow-influenced stabilization of liquid columns. J. Colloid Interface Sci. 170 (1995) 38-43

[91] D. Lyubimov, T. Lyubimova, A. Croell, P. Dold, K. Benz and B. Roux, Vibration-induced convective flows. Microgravity Sci. Technol. 11 (1998) 101-106.

[92] D. Lyubimov, T. Lyubimova and B. Roux, Mechanisms of vibrational control of heat transfer in a liquid bridge. Int. J. Heat Mass Transf. 40 (1997) 4031-4042.

[93] D.V. Lyubimov and A.A. Cherepanov, Development of a steady relief at the interface of fluids in a vibrational field. Fluid Dyn. Res. 21 (1986) 849-854.

[94] D.V. Lyubimov, A.A. Cherepanov, T.P. Lyubimova and B. Roux, Deformation of gas or drop inclusion in high frequency vibrational field. Microgravity Q. 6 (1996) 69-73.

[95] D.V. Lyubimov, A.A. Cherepanov, T.P. Lyubimova and B. Roux, Interface orienting by vibration. C. R. Acad. Sci. Paris, Ser. IIb 325 (1997) 391-396.

[96] D.V. Lyubimov, A.O. Ivantsov, T.P. Lyubimova and G.L. Khilko, Numerical modeling of frozen wave instability in fluids with high viscosity contrast. Fluid Dyn. Res. 48 (2016) 061415.

[97] D.V. Lyubimov, T.P. Lyubimova and A.A. Cherepanov, Dynamics of Interfaces in Vibrational Fields, Fizmatlit, (2003), in Russian.

[98] T. Lyubimova, A. Ivantsov, Y. Garrabos, C. Lecoutre and D. Beysens, Faraday waves on band pattern under zero gravity conditions. Phys. Rev. Fluids 4 (2019) 064001.

[99] T. Lyubimova, A. Ivantsov, Y. Garrabos, C. Lecoutre, G. Gandikota and D. Beysens, Band instability in near-critical fluids subjected to vibrationunder weightlessness. Phys. Rev. E 95 (2017) 013105.

[100] T.P. Lyubimova, R.V. Scuridin, A. Cröll and P. Dold, Influence of high frequency vibrations on fluid flow and heat transfer in a floating zone. Crys. Res. Technol. 38 (2003) 635-653.

[101] S. Madruga and C. Mendoza, Heat transfer performance and melting dynamic of a phase change material subjected to thermocapillary effects. Int. J. Heat Mass Transf. 109 (2017) 501-510.

[102] A. Manela and I. Frankel, On the Rayleigh-Bénard problem: dominant compressibility effects. J. Fluid Mech. 565 (2006) 461-475.

[103] C. Marangoni, Sull'espansione delle goccie d'un liquido galleggianti sulla superfice di altro liquido, Fratelli Fusi, (1865).

[104] M.J. Marr-Lyon, D.B. Thiessen and P.L. Marston, Stabilization of a cylindrical capillary Rayleigh-Plateau limit using acoustic radiation pressure and active feedback. J. Fluid Mech. 351 (1997) 345-357.

[105] G. Martin, S. Hoath and I. Hutchings, Inkjet printing - The physics of manipulating liquid jets and drops. J. Phys. Conf. Ser. 105 (2008) 012001.

[106] A. Mialdun, I.I. Ryzhkov, D.E. Melnikov and V. Shevtsova, Experimental evidence of thermal vibrational convection in a nonuniformly heated fluid in a reduced gravity environment. Phys. Rev. Lett. 101 (2008) 084501. 
[107] A.B. Mikishev and A.A. Nepomnyashchy, Large-scale Marangoni convection in a liquid layer with insoluble surfactant under heat flux modulation. J. Adhes. Sci. Technol. 25 (2011) 1411-1423.

[108] J. Miles and D. Henderson. Parametrically forced surface waves. Annu. Rev. Fluid Mech. 22 (1990) $143-165$.

[109] A.I. Mizev and D. Schwabe, Convective instabilities in liquid layers with free upper surface under the action of an inclined temperature gradient. Phys. Fluids 21 (2009) 112102.

[110] J. Moehlis, J. Porter and E. Knobloch, Heteroclinic dynamics in a model of Faraday waves in a square container. Physica D 238 (2009) 846-859.

[111] F. Moisy, G.-J. Michon, M. Rabaud and E. Sultan, Cross-waves induced by the vertical oscillation of a fully immersed vertical plate. Phys. Fluids 24 (2012) 022110.

[112] F. Muldoon, Numerical study of hydrothermal wave suppression in thermocapillary flow using a predictive control method. Comput. Math. Math. Phys. 58 (2018) 493-507.

[113] S. Paolucci and D.R. Chenoweth, Departures from the Boussinesq approximation in laminar Bénard convection. Phys. Fluids 30 (1987) 1561-1564.

[114] H.M. Park, M.C. Sung and J.S. Chung, Stabilization of Rayleigh-Bénard convection by means of mode reduction. Proc. R. Soc. A 460 (2004) 1807-1830.

[115] M. Pastoor, L. Henning, B.R. Noack, R. King and G. Tadmor, Feedback shear layer control for bluff body drag reduction. J. Fluid Mech. 608 (2008) 161-196.

[116] J.R.A. Pearson, On convection cells induced by surface tension. J. Fluid Mech. 4 (1958) 489-500.

[117] J.M. Perez-Gracia, J. Porter, F. Varas and J.M. Vega, Oblique cross-waves in horizontally vibrated containers. Fluid Dyn. Res. 46 (2014) 041410.

[118] J.M. Perez-Gracia, J. Porter, F. Varas and J.M. Vega, Subharmonic capillary-gravity waves in large containers subject to horizontal vibrations. J. Fluid Mech. 739 (2014) 196-228.

[119] C.-T. Pham, S. Perrard and G. Le Doudic, Surface waves along liquid cylinders. Part 1. Stabilising effect of gravity on the Plateau-Rayleigh instability. J. Fluid Mech. 891 (2020) A8.

[120] J.A.F. Plateau, Statique experimentale et theorique des liquides soumis aux seules forces moleculaires. 2 (1873). GauthierVillars.

[121] J. Porter, I. Tinao, A. Laverón-Simavilla and C.A. Lopez. Pattern selection in a horizontally vibrated container. Fluid Dyn. Res. 44 (2012) 065501.

[122] J. Porter, I. Tinao, A. Laverón-Simavilla and J. Rodríguez, Onset patterns in a simple model of localized parametric forcing. Phys. Rev. E 88 (2013) 042913.

[123] J. Porter, C.M. Topaz and M. Silber, Pattern control via multifrequency parametric forcing. Phys. Rev. Lett. 93 (2004) 034502.

[124] O. Pouliquen, J.M. Chomaz and P. Huerre, Propagating Holmboe waves at the interface between two immiscible fluids. J. Fluid Mech. 266 (1994) 277-302.

[125] D.S. Praturi and S.S. Girimaji, Mechanisms of canonical Kelvin-Helmholtz instability suppression in magnetohydrodynamic flows. Phys. Fluids 31 (2019) 024108.

[126] F. Preisser, D. Schwabe and A. Scharmann, Steady and oscillatory thermocapillary convection in liquid columns with free cylindrical surface. J. Fluid Mech. 126 (1983) 545-567.

[127] B. Protas and T. Sakajo, Harnessing the Kelvin-Helmholtz instability: feedback stabilization of an inviscid vortex sheet. $J$. Fluid Mech. 852 (2018) 146-177.

[128] Lord Rayleigh, Investigation of the character of the equilibrium of an incompressible heavy fluid of variable density. Proc. London Math. Soc. s1-14 (1882) 170-177.

[129] Lord Rayleigh, On convection currents in a horizontal layer of fluid, when the higher temperature is on the under side. Phil. Mag., Ser.6 32 (1916) 529-546.

[130] Lord Rayleigh Sec. R.S. XIX. On the instability of cylindrical fluid surfaces. London, Edinburgh Dublin Philos. Mag. J. Sci. 34 (1892) (207) 177-180.

[131] A.M. Rucklidge, M. Silber and A.C. Skeldon, Three-wave interactions and spatiotemporal chaos. Phys. Rev. Lett. 108 (2012) 074504 .

[132] P. Salgado Sánchez, J. Fernández, I. Tinao and J. Porter, Vibroequilibria in microgravity: Comparison of experiments and theory. Phys. Rev. E 100 (2019) 063103.

[133] P. Salgado Sánchez, Y. Gaponenko, V. Yasnou, A. Mialdun, J. Porter and V. Shevtsova, Effect of initial interface orientation on patterns produced by vibrational forcing in microgravity. J. Fluid Mech. 884 (2020) A38.

[134] P. Salgado Sánchez, Y.A. Gaponenko, J. Porter and V. Shevtsova, Finite-size effects on pattern selection in immiscible fluids subjected to horizontal vibrations in weightlessness. Phys. Rev. E 99 (2019) 042803.

[135] P. Salgado Sánchez, J. Porter, I. Tinao and A. Laverón-Simavilla, Dynamics of weakly coupled parametrically forced oscillators. Phys. Rev. E 94 (2016) 022216.

[136] P. Salgado Sánchez, V. Yasnou, Y. Gaponenko, A. Mialdun, J. Porter and V. Shevtsova, Interfacial phenomena in immiscible liquids subjected to vibrations in microgravity. J. Fluid Mech. 865 (2019) 850-883.

[137] P. Salgado Sánchez, J.M. Ezquerro, J. Fernández and J. Rodriguez, Thermocapillary effects during the melting of phase change materials in microgravity: Heat transport enhancement. Int. J. Heat Mass Transf. 163 (2020) 120478.

[138] P. Salgado Sánchez, J.M. Ezquerro, J. Fernández and J. Rodríguez, Thermocapillary effects during the melting of phase-change materials in microgravity: steady and oscillatory flow regimes. J. Fluid Mech. 908 (2021) A20. 
[139] P. Salgado Sánchez, J.M. Ezquerro, J. Porter, J. Fernández and I. Tinao, Effect of thermocapillary convection on the melting of phase change materials in microgravity: Experiments and simulations. Int. J. Heat Mass Transf. 154 (2020) 119717.

[140] A.E. Samoilova and A. Nepomnyashchy, Nonlinear feedback control of Marangoni wave patterns in a thin film heated from below. Physica D 412 (2020) 132627.

[141] R. Sattler, S. Gier, J. Eggers and C. Wagner, The final stages of capillary break-up of polymer solutions. Phys. Fluids 24 (2012) 023101.

[142] H.A. Schäffer, Second-order wavemaker theory for irregular waves. Ocean Eng. 23 (1996) 47-88.

[143] D. Schwabe, Thermocapillary liquid bridges and Marangoni convection under microgravity-Results and lessons learned. Microgravity Sci. Technol. 26 (2014) 1-10.

[144] D. Schwabe and A. Scharmann, Some evidence for the existence and magnitude of a critical Marangoni number for the onset of oscillatory flow in crystal growth melts. J. Cryst. Growth 46 (1979) 125-131.

[145] D. Sharp, An overview of Rayleigh-Taylor instability. Physica D 12 (1984) 3-18.

[146] M. Sheldrake and R. Sheldrake, Determinants of Faraday wave-patterns in water samples oscillated vertically at a range of frequencies from 50-200 Hz. Water 9 (2017) 1-27.

[147] V. Shevtsova, Y. Gaponenko, H. Kuhlmann, M. Lappa, M. Lukasser, S. Matsumoto, A. Mialdun, J. Montanero, K. Nishino and I. Ueno, The JEREMI-project on thermocapillary convection in liquid bridges. Part B: Overview on impact of co-axial gas flow. Fluid Dyn. Mater. Process. 10 (2014) 197-240.

[148] V. Shevtsova, Y. Gaponenko and A. Nepomnyashchy, Thermocapillary flow regimes and instability caused by a gas stream along the interface. J. Fluid Mech. $\mathbf{7 1 4}$ (2013) 644-670.

[149] V. Shevtsova, Y.A. Gaponenko, V. Yasnou, A. Mialdun and A. Nepomnyashchy, Two-scale wave patterns on a periodically excited miscible liquid-liquid interface. J. Fluid Mech. 795 (2016) 409-422.

[150] V. Shevtsova, I.I. Ryzhkov, D.E. Melnikov, Y.A. Gaponenko and A. Mialdun, Experimental and theoretical study of vibrationinduced thermal convection in low gravity. J. Fluid Mech. 648 (2010) 53-82.

[151] J. Shiomi, M. Kudo, I. Ueno, H. Kawamura and G. Amberg, Feedback control of oscillatory thermocapillary convection in a half-zone liquid bridge. J. Fluid Mech. 496 (2003) 193-211.

[152] F. Simonelli and J.P. Gollub, Surface wave mode interactions: Effects of symmetry and degeneracy. J. Fluid Mech. 199 (1989) 349-354.

[153] W.A. Sirignano and I. Glassman, Flame spreading above liquid fuels: Surface tension driven flows. Combust. Sci. Technol. 1 (1970) 307.

[154] A.C. Skeldon and J. Porter, Scaling properties of weakly nonlinear coefficients in the Faraday problem. Phys. Rev. E 84 (2011) 016209.

[155] L.A. Slobozhanin and J.M. Perales, Stability of liquid bridges between equal disks in an axial gravity field. Phys. Fluids A $\mathbf{5}$ (1993) 1305-1314.

[156] W.D. Smyth, G.P. Klaassen and W.R. Peltier, Finite amplitude holmboe waves. Geophys. Astrophys. Fluid Dyn. 43 (1988) 181-222.

[157] W.D. Smyth and W.R. Peltier, Instability and transition in finite-amplitude Kelvin-Helmholtz and Holmboe waves. J. Fluid Mech. 228 (1991) 387-415.

[158] J.W. Strutt, VI. On the capillary phenomena of jets. Proc. R. Soc. London 29 (1879) 71-97.

[159] R.S. Subramanian and R. Balasubramanian, The Motion of Bubbles and Drops in Reduced Gravity. Cambridge University Press, Cambridge (2001).

[160] A. Swaminathan, S. Garrett, M. Poese and R. Smith, Dynamic stabilization of the Rayleigh-Bénard instability by acceleration modulation. J. Acoust. Soc. Am. 144 (2018) 2334-2343.

[161] S. Taneda, Visual observations of the flow around a half-submerged oscillating circular cylinder. Fluid Dyn. Res. 13 (1994) 119-151.

[162] G.I. Taylor, The instability of liquid surfaces when accelerated in a direction perpendicular to their planes. I. Proc. R. Soc. A 201 (1950) 192-196.

[163] U. Thiele, J.M. Vega and E. Knobloch, Long-wave Marangoni instability with vibration. J. Fluid Mech. 546 (2006) 61-87.

[164] S.A. Thorpe, Experiments on the instability of stratified shear flows: miscible fluids. J. Fluid Mech. 46 (1971) $299-319$.

[165] I. Tinao, J. Porter, A. Laverón-Simavilla and J. Fernández, Cross-waves excited by distributed forcing in the gravity-capillary regime. Phys. Fluids 26 (2014) 024111.

[166] C.M. Topaz, J. Porter and M. Silber, Multifrequency control of Faraday wave patterns. Phys. Rev. E 73 (2004) 066206.

[167] C.M. Topaz and M. Silber, Resonances and superlattice pattern stabilization in two-frequency forced Faraday waves. Physica D 172 (2002) 1-29.

[168] M. Troitiño, P. Salgado Sánchez, J. Porter and D. Gligor, Symmetry breaking in large columnar frozen wave patterns in weightlessness. Microgravity Sci. Technol. 32 (2020) 907-919.

[169] A.M. Turing, The chemical basis of morphogenesis. Phil. Trans. R. Soc. Lond. B 237 (1952) 37-72.

[170] L. Turyn, The damped Mathieu equation. Q. Appl. Math. 51 (1993) 389-398.

[171] W.B. Underhill, S. Lichter and A.J. Bernoff, Modulated, frequency-locked and chaotic cross-waves. J. Fluid Mech. 225 (1991) 371-394.

[172] F. Varas and J.M. Vega, Modulated surface waves in large-aspect-ratio horizontally vibrated containers. J. Fluid Mech. 579 (2007) 271-304. 
[173] J.T. Waddell, C.E. Niederhaus and J.W. Jacobs, Experimental study of Rayleigh-Taylor instability: Low Atwood number liquid systems with single-mode initial perturbations. Phys. Fluids 13 (2001) 1263-1273.

[174] J. Walker, L.M. Witkowski and B. Houchens, Effects of a rotating magnetic field on the thermocapillary instability in the floating zone process. J. Cryst. Growth 252 (2003) 413-423.

[175] G.H. Wolf, The dynamic stabilization of the Rayleigh-Taylor instability and the corresponding dynamic equilibrium. $Z$. Physik 227 (1969) 291-300.

[176] G.H. Wolf, Dynamic stabilization of the interchange instability of a liquid-gas interface. Phys. Rev. Lett. 24 (1970) $444-446$.

[177] V. Yasnou, Y. Gaponenko, A. Mialdun and V. Shevtsova, Influence of a coaxial gas flow on the evolution of oscillatory states in a liquid bridge. Int. J. Heat Mass Transf. 123 (2018) 747-759.

[178] S. Zen'kovskaya, V. Novosyadlyi and A. Shleikel', The effect of vertical vibration on the onset of thermocapillary convection in a horizontal liquid layer. J. Appl. Math. Mech. 71 (2007) 247-257.

[179] W. Zhang and J. Viñals, Pattern formation in weakly damped parametric surface waves. J. Fluid Mech. 336 (1997) 301-330. 